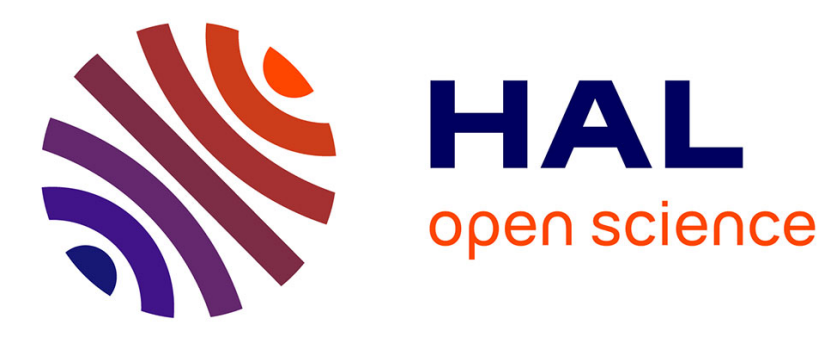

\title{
La fortification du Bas-Empire
}

Gertrud Kuhnle, Alexandre Burgevin, Bérangère Fort, Jean-Pierre Mazimann, Sylvie Mouton-Venault

\section{To cite this version:}

Gertrud Kuhnle, Alexandre Burgevin, Bérangère Fort, Jean-Pierre Mazimann, Sylvie Mouton-Venault. La fortification du Bas-Empire. Gallia - Archéologie de la France antique, 2007, Epomanduodurum, une ville chez les Séquanes: bilan de quatre années de recherche à Mandeure et Mathay (Doubs), 64, pp.407-426. 10.3406/galia.2007.3322 . halshs-00610901

\section{HAL Id: halshs-00610901 https://shs.hal.science/halshs-00610901}

Submitted on 7 Feb 2020

HAL is a multi-disciplinary open access archive for the deposit and dissemination of scientific research documents, whether they are published or not. The documents may come from teaching and research institutions in France or abroad, or from public or private research centers.
L'archive ouverte pluridisciplinaire HAL, est destinée au dépôt et à la diffusion de documents scientifiques de niveau recherche, publiés ou non, émanant des établissements d'enseignement et de recherche français ou étrangers, des laboratoires publics ou privés.

\section{(ㅇ)(1) $\$$}

Distributed under a Creative Commons Attribution - NonCommercial - NoDerivatives| 4.0 
dans la ville antique et constituer, probablement, le repère monumental majeur du paysage urbain.

Les prospections menées depuis 2001 sur l'ensemble de l'agglomération ont considérablement enrichi la parure monumentale de la ville antique, en particulier dans le secteur du théâtre qui se voit désormais doté d'une riche série d'édifices publics (fig. 4 et pl. X, hors texte). Comme à Avenches, l'esplanade qui séparait le sanctuaire de l'édifice de spectacle est encadrée par toute une série de constructions qui ont été révélées aussi bien au sud qu'au nord du théâtre. Seule une fouille permettra de les identifier plus précisément, mais on devine déjà des lieux de cultes secondaires ou des édifices nécessaires pour l'accueil ou le confort des pèlerins. D'autres grands bâtiments se laissent entrevoir dans le centre de l'agglomération, dans la courbe du Doubs par exemple. Ce constat d'un riche équipement architectural, que l'on pouvait déjà faire au vu des découvertes anciennes par F. Morel-Macler d'un lapidaire abondant attestant l'existence de plusieurs constructions monumentales, a donc été confirmé par les prospections réalisées depuis trois ans. Si l'on ajoute à ces observations que les premières études menées sur la décoration architecturale ont établi qu'il faut restituer aux monuments publics une décoration architecturale riche et soignée, on conviendra que nous avons probablement affaire avec ce site à une métropole urbaine qui présente tous les attributs de l'urbanitas romaine. Reste à ajouter que le théâtre a probablement joué un rôle directeur dans la mise en place de ce paysage monumental: on a pu en effet remarquer que bon nombre des édifices repérés en prospection, à proximité du théâtre bien sûr mais également dans la courbe du Doubs, sont alignés sur celui-ci, comme si l'équipement architectural s'était trouvé aimanté en quelque sorte par l'édifice de spectacle.

Ces premiers résultats confirment pleinement l'impression que donnaient déjà, outre les dimensions considérables de la ville antique, 180 ha urbanisés, le grand sanctuaire à péribole ovale et temple périptère sine postico, l'établissement thermal de Courcelles (Mougin, Watts, 1997), l'inscription évergétique de Catullus (CIL, XIII, 5416 a et b, et 5417), la présence du culte impérial, la statue de Mars (Pichot, 1995a et b), etc. : Epomanduodurum était une importante ville gallo-romaine. Il n'est pas question de discuter ici du statut de cette ville. Mandeure était, au sens juridique du terme, une agglomération secondaire, mais son épiderme monumental et son importance dans la géographie urbaine de la région étaient, quant à eux, loin d'être secondaires. Il convient d'insister sur ce point pour s'inscrire en faux contre une synthèse publiée très récemment qui pourrait laisser penser que Mandeure n'était qu'une simple bourgade à peine dotée des éléments de composantes urbaines (Walter, 2003). Un rapide coup d'œil aux plans les plus récents de la ville antique ou même une simple visite sur le site du théâtre suffisent à démentir complètement de telles conclusions, il est vrai, écrites avant le début de nos travaux.

\section{CONCLUSION}

La reprise des recherches sur le théâtre antique de Mandeure, recherches qui se poursuivront dans les années qui viennent pour aboutir à une publication monographique, s'inscrit dans un contexte de reprise des études sur les théâtres antiques des provinces de Gaule ou de Germanie (Dumasy, 2000). Les édifices de spectacle d'Augst et d'Avenches, pour ne citer que deux exemples très proches géographiquement de Mandeure, ont fait l'objet de travaux importants ces dernières années ${ }^{17}$. Il était temps car le débat sur la forme architecturale du théâtre gallo-romain et sa fonction commençait à «tourner en rond » (Dumasy, 1989). On sait que la plupart des édifices de spectacle ont souvent été fouillés avant la Première Guerre mondiale, la grande majorité entre 1850 et 1900 . C'est dire que la documentation ne s'est guère renouvelée depuis A. de Caumont, le premier qui regroupa les théâtres des provinces du nordouest de l'Empire dans cette catégorie à la fois topique et fonctionnelle. Depuis les années 1960, les fouilles ou la reprise des dégagements des théâtres d'Alésia, de Grand, de Ribemont-sur-Ancre (Cadoux, 1992), de Canouville (Le Maho, Nicolle, 1979), de Vendeuil-Caply (Dufour, 1992), de Lenzburg (Niffeler, 1988), de Ladenburg (Filzinger dir., 1976) ou de Dalheim (Krier, Wagner, 1985), attestent certes un regain d'intérêt pour les édifices de spectacle dans les Gaules et les Germanies, mais on déplore toujours l'absence de véritables publications monographiques.

J.-Y. M., P. M., S. B., N. A.

\section{LA FORTIFICATION DU BAS-EMPIRE}

Notre premier bilan ne repose qu'en partie sur les acquis de deux campagnes de fouilles, réalisées dans le cadre du PCR en 2002 et 2003. Ils constituent, en fonction du programme du PCR 2001-2004, avant tout les résultats tirés de l'analyse et de l'exploitation d'un grand nombre de données et d'objets récoltés au fil des années par de

17. Pour Augst: Schwarz, 1991 et Horisberger, 1997 (avec la bibliographie des dernières campagnes). Pour Avenches : Matter, 1999 et Bridel, 2004. 
nombreux chercheurs et autres passionnés. La majorité des données concernant Mandeure au Bas-Empire provient de l'emprise de la fortification. L'existence de cette dernière resta inconnue, même au moment des investigations anciennes menées dans les proches ruines du théâtre et du sanctuaire. Sa présence ne fut supposée qu'au milieu $\mathrm{du} \mathrm{XX}^{\mathrm{e}} \mathrm{s}$. et confirmée par une série de sondages réalisés à la fin des années 1990. Avec la mise au jour d'une partie de son enceinte, en 2002 et 2003, nous avons pu obtenir des certitudes en ce qui concerne son tracé et des précisions au sujet de son architecture. L'enceinte, munie de tours saillantes demi-circulaires, a la forme d'une cloche qui s'appuie sur le Doubs. Par conséquent, les similitudes entre la fortification de Mandeure et celles connues sur l'Aare (Suisse), à Soleure ou à Olten par exemple, sont grandes.

Il est important de souligner que nous adoptons de manière délibérée et exclusive le terme de «fortification », de loin le mieux approprié. En effet, le mot «fortin », qui a pu être utilisé anciennement, renvoie à une image fausse de l'ouvrage. D'autre part, en l'absence de preuves formelles, il est trop tôt pour employer à juste titre les termes latins castrum ou castellum.

\section{PROBLÉMATIQUE DE LA RECHERCHE}

Après le retrait des militaires romains du limes germanorhétique dans la seconde moitié du $\operatorname{III}^{\mathrm{e}} \mathrm{s}$. de notre ère, l'agglomération romaine de Mandeure ne se trouve plus qu'à une soixantaine de kilomètres de la nouvelle frontière rhénane de l'Empire. Située sur un axe de communication important, à mi-chemin entre Vesontio/Besançon, ancienne capitale de la cité des Séquanes, promue capitale de la province de Maxima Sequanorum vers la fin du III $^{\mathrm{e}}$ s., et Cambete/Kembs au bord du Rhin, l'intérêt stratégique de Mandeure paraît évident aussi bien sur le plan militaire que commercial. Les dynamiques urbaines ne sont plus les mêmes que sous le Haut-Empire, mais les facteurs de localisation n'ont guère changé ; la situation particulière de Mandeure reste un critère essentiel dans la nouvelle carte géopolitique qui se met en place après la « crise » du III $^{\mathrm{e}} \mathrm{s}$. L'agglomération est toujours implantée au bord d'un coude du Doubs, à un point de rupture de charge. C'est très probablement à cet endroit que les marchandises, acheminées jusqu'ici par voie d'eau (Rhône-Saône-Doubs), devaient être déchargées pour pouvoir être transportées ensuite par voie terrestre, passant entre les Hautes Vosges et l'arc jurassique (trouée de Belfort) vers les agglomérations ou les établissements militaires s'échelonnant le long du Rhin.

On comprend aisément, pour toutes ces raisons, que l'agglomération qui revêtait au Haut-Empire les caractéristiques d'une véritable ville (plus de 180 ha urbanisés répartis sur 300 ha), à la parure monumentale exceptionnelle, se soit transformée en site urbain fortifié. De taille plutôt modeste par rapport à l'étendue de l'agglomération du Haut-Empire puisque l'enceinte ne circonscrit qu'environ 1,5 ha, cette fortification perpétuait probablement le pouvoir et le prestige d'Epomanduodurum au-delà du III $^{\mathrm{e}} \mathrm{s}$. Le site fortifié assurait certainement la protection d'un port fluvial et peut-être aussi celle d'un pont sur le Doubs.

C'est à la spoliation des édifices publics et au réemploi des matériaux de démolition de ces derniers dans un nouveau programme d'urbanisme, dont fait partie la fortification, que nous devons, pour l'essentiel, la conservation des éléments architecturaux de la ville du Haut-Empire. La destruction d'anciens édifices publics et la construction de la fortification, toutes deux réalisées sous une autorité forte (au moins municipale pour la première action et vraisemblablement impériale en ce qui concerne la seconde), ont modifié en profondeur la trame urbaine d'Epomanduodurum et entraîné un nouveau dynamisme, dont quelques points forts peuvent être dégagés.

Dans le présent bilan, nous nous intéressons dans un premier temps à la topographie, la forme et l'architecture de l'ouvrage à travers les indices anciens et les données récentes. L'examen synthétique des principales catégories de mobilier archéologique permet ensuite de compléter l'image de la fortification en ce qui concerne notamment sa chronologie et sa fonction.

G. K.

\section{LOCALISATION DE LA FORTIFICATION DU BAS-EMPIRE}

La fortification tardive occupe une position assez centrale. Elle se trouve, par exemple, à distance égale (environ $2 \mathrm{~km}$ ) des thermes de Courcelles (Mandeure) et du quartier artisanal de l'Essarté (Mathay). Située sur la rive droite du Doubs, au lieu-dit le Clos du Château, à moins de $400 \mathrm{~m}$ du théâtre et du sanctuaire, elle est orientée vers le nord-ouest, côté rivière (fig. 2).

G. K. 


\section{LES INTERVENTIONS ANTÉRIEURES AUX CAMPAGNES DU PROJET COLLECTIF DE RECHERCHE}

Malgré l'ancienneté des recherches archéologiques menées sur l'antique Epomanduodurum, la découverte de la fortification du Bas-Empire est très récente. Cela tient bien sûr à la nature des vestiges correspondant, les fouilles anciennes ne s'intéressant qu'aux vestiges de maçonneries romaines «classiques». C'est l'architecte F. Morel-Macler qui au début du XIX ${ }^{\mathrm{e}} \mathrm{s}$. fut le premier à lever les vestiges antiques de Mandeure. Outre le théâtre et quelques murs, il releva l'emplacement d'un grand nombre d'amas pierreux issus des récupérations et appelés murgers (Golbéry, 1828, pl. 4 ; Morel-Macler, 1847, pl. 2) (fig. 3). Ces vestiges d'épierrements, qui nous renseignent sur la localisation des murs antiques victimes de spoliations, furent répertoriés et cartographiés, au même titre que les vestiges et les découvertes archéologiques traditionnelles; mais rien, dans tout ce qu'il mit au jour dans le secteur de la fortification, ne signalait à ses yeux une occupation du BasEmpire. On verra plus bas l'importance de ces murgers, dont l'inventeur ne saisit pas la signification. Quant aux autres vestiges découverts alors, ils correspondent tous au Haut-Empire ${ }^{18}$.

En 1893, P. Barbier réalisa, à l'initiative de la Société d'émulation de Montbéliard, un plan du site archéologique de Mandeure-Mathay (archives du musée de Montbéliard, quatre feuilles A2). Il avait à sa disposition le levé du géomètre Roland, dit improprement «cadastre napoléonien ». P. Barbier reporta sur ce fond cadastral toutes les données du plan de F. Morel-Macler, rajouta les édifices, sanctuaire et théâtre, dégagés dans la seconde moitié du

18. F. Morel-Macler produisit deux plans qui ne sont pas tout à fait identiques : le premier fut publié dansl'Annuaire du Doubsen 1822, p. 124, et le second par P. de Golbéry en 1828 (Golbéry, 1928, pl. 4). C'est le second plan, intitulé Carte topographique du villagede Mandeureet de ses environs avec indication des Ruines Romaines que F. Morel-Macler publia en 1847 dans son ouvrage sur les Antiquités de Mandeure (Morel-Macler, 1847, pl. 2). Si l'emplacement des murgers est quasi identique sur les deux plans, ce n'est pas le cas pour les autres vestiges: - sur le plan de 1822, un "Portique» est mentionné non loin de la rive droite du Doubs. En face, sur la rive gauche du cours d'eau, est indiquée la "Culée d'un pont»; - sur le plan de 1828-1847, ce sont les points 10, 11 et 12, légendés respectivement "Porte triomphale déc. en 1783-1836-1837-1844", "Temple de Jupiter Ammon » et « Culée de pont », qui peuvent, par leur localisation, être mis en relation avec le site de la fortification tardive. La "Culée d'un pont", l'unique vestige mentionné sur les deux plans, est localisée d'abord à $80 \mathrm{~m}$ au sud-ouest de l'actuel pont (plan de 1822), puis à l'emplacement de ce dernier (plan de 1828-1847: $\left.\mathrm{n}^{\circ} 12\right)$.
XIX $^{\mathrm{e}}$ s. et compléta le document par le levé de nombreux vestiges rendus visibles par l'importante sécheresse de 1893.

On constate que le lieu-dit occupé par la fortification tardive s'appelle, dès la fin du XIX ${ }^{\mathrm{e}}$ s., Champs des Cloux du Château. Outre les murgers, cinq découvertes y sont mentionnées, mais aucune n'est attribuée à une fortification tardive ${ }^{19}$ (fig. 3 et pl. XIV, hors texte).

Entre 1950 et 1960, des découvertes fortuites furent réalisées à l'occasion de la construction d'habitations privées de part et d'autre de la rue du Pont. Parmi elles, on compte une fibule cruciforme trouvée sur l'une des quatre parcelles bâties au nord de la rue (n ${ }^{\text {os }} 134-136-138-140$, rue du Pont) et surtout plusieurs monnaies provenant des maisons Jacquets, situées au sud de la rue du Pont ( $\mathrm{n}^{\text {os }} 1 \mathrm{bis-}$ 3-5, impasse du Clos). Ces trouvailles sont datées du IV ${ }^{\mathrm{e}} \mathrm{s}$. (Mazimann, 1992).

C'est Y. Jeannin qui, dans les années 1960, émit l'hypothèse de l'existence d'une fortification tardive. En effet, il établit une corrélation entre le nom du lieu-dit le Clos du Château, qui ne s'explique par aucun vestige médiéval, le fait que Mandeure était qualifié de «castrum» au haut Moyen Âge, la découverte d'objets du IV ${ }^{\mathrm{e}} \mathrm{s}$. dans ce secteur et une anomalie du parcellaire figurant sur le cadastre napoléonien (les parcelles affectent un arc de cercle en bordure du Doubs) qui évoque une fortification en forme de cloche s'appuyant sur le cours d'eau (Jeannin, 1966 et 1986).

En 1984, Y. Jeannin eut l'occasion de vérifier son hypothèse en suivant, dans le cadre d'une fouille de sauvetage, la tranchée du gazoduc (pl. XII, hors texte). Deux sondages sur les cinq réalisés se situent dans l'emprise hypothétique

19. Les découvertes suivantes sont reportées sur le plan : - un alignement de quatre colonnes, orienté nord-est/sud-ouest, annoté «PORTE TRIOMPHALE 1783 ». Il est curieux que P. Barbier associe cette légende au «Portique» du premier plan de F. MorelMacler (1822) alors que le point 10, appelé sur le second plan de F. Morel-Macler "Porte triomphale", se situe à une cinquantaine de mètres au nord-est de l'alignement des quatre colonnes; - une petite croix de localisation marquée «APOLLON" : il s'agit d'une statuette en bronze (musée de Montbéliard, no d'inv. 2191) qui fut découverte en 1894 et que C. Lalance décrivit dans sa notice (Lalance, 1895) en spécifiant que le point de fouille avait été rajouté avec précision sur le plan dressé en 1893 par P. Barbier ; - l'indication de «TEMPLE DE JUPITER AMMON 1781 », sans localisation précise au sein de l'emprise estimée de la fortification; - l'indication «STATUE DE JUPITER 1781 » à l'est de l'emprise supposée de l'enceinte : il s'agit en fait d'une statue colossale de Mars dont les fragments ont été trouvés dans un des murgers du lieu-dit Champs des Fougères (Pichot, 1995a et b) ; - une maçonnerie formant un U, légendée "Ancienne culée d'un Pont ", sur la rive gauche du Doubs. 
de la fortification tardive (sondages A et B). Il découvrit à $45 \mathrm{~m}$ de la culée du pont actuel, le bord d'un empierrement long de $6 \mathrm{~m}$ (sondage $\mathrm{A}$ ) et, à $120 \mathrm{~m}$, un massif de mortier et des blocs entassés de façon à former une sorte de mur long d'environ $12 \mathrm{~m}$, orienté obliquement en direction du Doubs (sondage B). Y. Jeannin pense avoir affaire, dans le sondage $\mathrm{B}$, à une digue refoulant le cours du Doubs pour éviter de saper la berge devant la fortification. Quant à l'empierrement remarqué dans le sondage A, il correspond à un aménagement antique indéterminé qui se situe à peu près dans l'axe de la fortification et dans lequel l'auteur propose de voir le blocage éventuel d'une pile de pont en bois.

J.-P. Mazimann publia en 1992, avec la collaboration de P. Mougin, une première synthèse portant sur l'occupation tardive de l'agglomération antique, à travers l'étude des monnaies, des molettes d'Argonne et d'autres objets retrouvés à l'occasion de prospections de surface sur le site fortifié et ses environs (Mazimann, 1992, fig. 2).

En 1997, la Société Terra Nova fut chargée par le Service régional de l'archéologie de Franche-Comté de procéder à une prospection magnétique sur une surface de 1,6 ha au sud de la route nationale, sur le site présumé de la fortification tardive (Dabas, Aubry, 1997) (pl. XIII, hors texte).

Le document permit d'établir que le fossé de la fortification se développe selon une courbe régulière et que l'espace intérieur de celle-ci est subdivisé en un secteur bâti au nordouest et un secteur sans maçonneries notables au sud-est. Ce dernier correspond à une très large voie orientée nordest/sud-ouest. Perpendiculairement à celle-ci se dessine au nord-ouest un îlot bâti mesurant environ $60 \mathrm{~m}$ dans le sens parallèle à la voie et au moins $80 \mathrm{~m}$ dans le sens perpendiculaire. Au nord-est de cet îlot, se distingue un axe très marqué, perpendiculaire à la rivière mais décalé par rapport à l'îlot. Par conséquent, il est fort probable que cet axe fonctionne avec la fortification du Bas-Empire. En revanche, le tracé exact du mur d'enceinte resta difficile à établir sur ce document aussi longtemps qu'il n'y eut pas de données de fouille qu'on aurait pu confronter aux anomalies de la carte de prospection magnétique (voir infra, p. 411-414).

En 1998, B. Chazaly réalisa un levé microtopographique sur les terrains accessibles au sud et au nord de la rue du Pont qui correspondent à l'emprise supposée de la fortification et à ses abords immédiats (pl. XIV, hors texte).

Ces données corroborent et complètent celles de la prospection magnétique. Au sud de la rue du Pont, on devine la moitié d'une plate-forme polygonale, circonscrite par un faisceau de courbes serrées qui inclut la courbe des $335 \mathrm{~m}$
NGF. En ce qui concerne ses dimensions maximales, cette demi-plate-forme, qui atteint une altitude absolue de $336 \mathrm{~m}$ NGF, mesure dans le sens perpendiculaire au Doubs, $140 \mathrm{~m}$ et, dans le sens parallèle au cours d'eau, $90 \mathrm{~m}$. La plateforme semble correspondre à la surface enclose par le mur d'enceinte.

Le levé microtopographique permet de situer aisément la berge actuelle du Doubs puisque l'altitude chute sur une distance de 12 à 20 m, de 335 m NGF à 333,70 m NGF. Mais la question est posée de savoir si cette berge correspond à la berge antique. Dans ce cas, l'alignement de quatre colonnes relevé en 1893 à l'emplacement d'un vestige que F. Morel-Macler avait appelé " portique » serait à mettre en relation avec un aménagement antique de berge. Même si la localisation concorde, nous devons rester prudents car nous ne disposons d'aucune information altimétrique en ce qui concerne ces vestiges. Par conséquent, nous ne pouvons pas exclure l'hypothèse selon laquelle la berge du Doubs se situait, dans l'Antiquité, plus près du cours d'eau.

En 1998, des fouilles de sauvetage, dites Béhra, ont été menées au 73, rue du Pont (parcelles 225 et 226) sous la responsabilité de J.-P. Mazimann et de P. Mougin (zone 1000) (pl. XII, hors texte).

En plan, les vestiges de l'Antiquité, bien que perturbés par plusieurs creusements modernes très étendus, révélaient une structuration de l'espace qui est marquée d'est en ouest par :

- la tranchée de fondation du mur d'enceinte tardif ;

- un vaste espace, large d'environ 20 m, interprété comme une cour ;

- la chaussée d'une voie en graviers, orientée nord-est/sudouest, large de $7 \mathrm{~m}$, pourvue de fosses qui ont été creusées dans l'ultime recharge conservée ;

- un espace initialement réservé à un hypothétique doublement de la bande de roulement de la voie et qui a finalement été occupé par des constructions légères sur poteaux ;

- un habitat avec portique, longeant la limite nord-ouest de l'espace réservé, qui a été plusieurs fois remanié entre le II ${ }^{\mathrm{e}}$ et le $\mathrm{IV}^{\mathrm{e}} \mathrm{s}$.

La même année, une première fouille programmée fut réalisée sous la direction de J.-P. Mazimann sur la parcelle 7 du cadastre: zone 2200 à 2700 (pl. XII, hors texte) (Mazimann dir., 1999).

Elle consistait en une tranchée discontinue, large de 2,40 $\mathrm{m}$ qui traversait, perpendiculairement au Doubs, toute l'emprise interne de la fortification. Orientée sud-est/nord-ouest, elle commence au bord du chemin 
d'exploitation $\mathrm{n}^{\circ} 19$ pour s'arrêter à une soixantaine de mètres de la rive du cours d'eau. Situé dans la zone prospectée en 1997, le tronçon le plus long (72 m) traverse l'espace bâti (zones 2200 à 2500) et l'espace dépourvu de maçonneries (zone 2600). Le tronçon qui se trouve près du chemin d'exploitation (zone 2700, 15 m de long) coupe le mur d'enceinte.

Dans l'espace bâti (zones 2200 à 2500), cinq murs ont pu être attribués au Haut-Empire et onze au Bas-Empire. Du Bas-Empire date également une série de trous de poteau aménagés dans la chaussée de la voie (zone 2600), déjà observée sur la fouille Béhra. Dans la zone 2700, la tranchée de fondation du mur d'enceinte coupe un angle maçonné, mais arasé, d'une construction indéterminée dont la couche de démolition a livré deux tessons de sigillée d'Argonne décorée à la molette, associés à une monnaie de Constant frappée à Lyon vers 340 (US 2715).

En 1999, deuxième année de fouille programmée, l'intervention réalisée sous la responsabilité de P. Mougin fut beaucoup plus limitée en raison d'un budget fortement réduit (Mougin, 2000). Il faut distinguer deux opérations (pl. XII, hors texte) :

- la fouille de la zone 3000 (tranchée longue d'environ $33 \mathrm{~m}$, large de $3,50 \mathrm{~m}$ ) a essentiellement permis d'identifier les niveaux du Haut-Empire et une tranchée de fondation large d'environ 2,70 m. Il pourrait s'agir du mur d'enceinte du côté du Doubs : quelques rares pierres de la semelle de fondation encore en place reposaient sur un pilotis de piquets (diamètre estimé entre $9 \mathrm{~cm}$ et $12 \mathrm{~cm}$ ), matérialisés par leurs trous disposés en quinconce tous les 20 à $30 \mathrm{~cm}^{20}$;

- cinq sondages (S. 1 à S. 5) ont été effectués au tractopelle pour confirmer ou infirmer la présence du fossé et du mur d'enceinte entre la zone 2700 et le Doubs. Leur faible largeur $(1 \mathrm{~m})$ et leur profondeur d'au moins $2 \mathrm{~m}$ ont contraint les archéologues à ne faire que des observations succinctes depuis le haut des tranchées sur des coupes non nettoyées. Par conséquent, ces sondages n'apportent que peu d'informations exploitables.

G. K.

\section{LES PRINCIPAUX APPORTS DES FOUILLES PROGRAMMÉES DE 2002 ET 2003}

Les deux campagnes de fouille en aire ouverte (zones 4000 et 4100), réalisées dans le cadre du projet collectif de recherche sur Mandeure, ont porté sur une superficie

20. Dispositif qui sera reconnu par la suite pour la fondation de l'enceinte du Bas-Empire. d'environ $1500 \mathrm{~m}^{2}$ (fig. 29 et 30). Elles concernaient une portion du front sud et sud-est de la fortification du BasEmpire, c'est-à-dire le tracé de l'enceinte et une partie du fossé. Les vestiges tardifs y affleurent directement sous la terre arable de faible épaisseur $(0,05 \mathrm{~m}$ à $0,30 \mathrm{~m})$.

Dans la zone étudiée, le substrat est formé de graviers et de galets mélangés à du sable jaune qui sont surmontés d'un limon d'origine alluvionnaire. La stratigraphie des couches d'occupation antérieures à la fortification atteint jusqu'à 0,90 $\mathrm{m}$ de haut. Le tronçon de l'enceinte étudié coupe, en biais, l'importante voie du Haut-Empire qui se dirige tout droit vers la zone de gué située face au sanctuaire (ce gué a pu être reconnu en 2003 par des recherches subaquatiques). La voie, matérialisée par une succession d'apports de graviers incluant parfois des couches à dominante limoneuse, atteint - bas-côtés inclus - un peu plus de $20 \mathrm{~m}$ de large. Elle est bordée au sud-est par un mur et au nordouest par un fossé latéral. À une dizaine de mètres au nord-ouest de la voie et toujours parallèle à celle-ci, a été rencontré un autre mur du Haut-Empire. Immédiatement au nord-ouest de ce dernier, une stratigraphie complexe témoigne d'une occupation dense du secteur (milieu du $\mathrm{I}^{\mathrm{er}} \mathrm{s}$. et fin $\mathrm{du} \mathrm{II}{ }^{\mathrm{e}} \mathrm{s}$.) correspondant à l'îlot bâti repéré par la prospection magnétique de 1997.

Les fouilles ont permis de mettre au jour, sur une longueur d'environ $85 \mathrm{~m}$, le tracé incurvé de l'enceinte (fig. 29). Sont conservées les fondations semi-circulaires de deux tours saillantes, de la courtine qui les relie et d'un segment de courtine qui se dirige vers le nord-est. Le fossé, distant de $8 \mathrm{~m}$ à $12 \mathrm{~m}$ de l'enceinte, est également incurvé. Ses dimensions, qui peuvent être estimées à environ $15 \mathrm{~m}$ de largeur et $3 \mathrm{~m}$ de profondeur, sont considérables.

La largeur de la tranchée de fondation du mur d'enceinte varie entre $3,20 \mathrm{~m}$ et $3,85 \mathrm{~m}$; sa profondeur conservée atteint $1,40 \mathrm{~m}$ entre les deux tours et près de $2 \mathrm{~m}$ dans le segment nord-est. Larges de $8 \mathrm{~m}$, les fondations semi-circulaires des tours sont saillantes de $4 \mathrm{~m}$ par rapport au front du mur d'enceinte. Entre les deux tours, le front extérieur de la tranchée de la courtine mesure précisément 39,69 m de long. Ainsi, les tours sont distantes de $50 \mathrm{~m}$, mesure prise entre les axes (fig. 29 et 31).

Dans la mesure où les fondations n'étaient pas entièrement spoliées, la technique de construction a pu être observée (fig. 31 à 33). La tranchée du mur d'enceinte ainsi que les excavations semi-circulaires des tours se caractérisent par des parois quasi verticales et un fond plat. Les fondations du mur et des tours reposent sur un radier de pieux battus qui a fait l'objet d'un examen dans un tronçon de 4,40 m, 


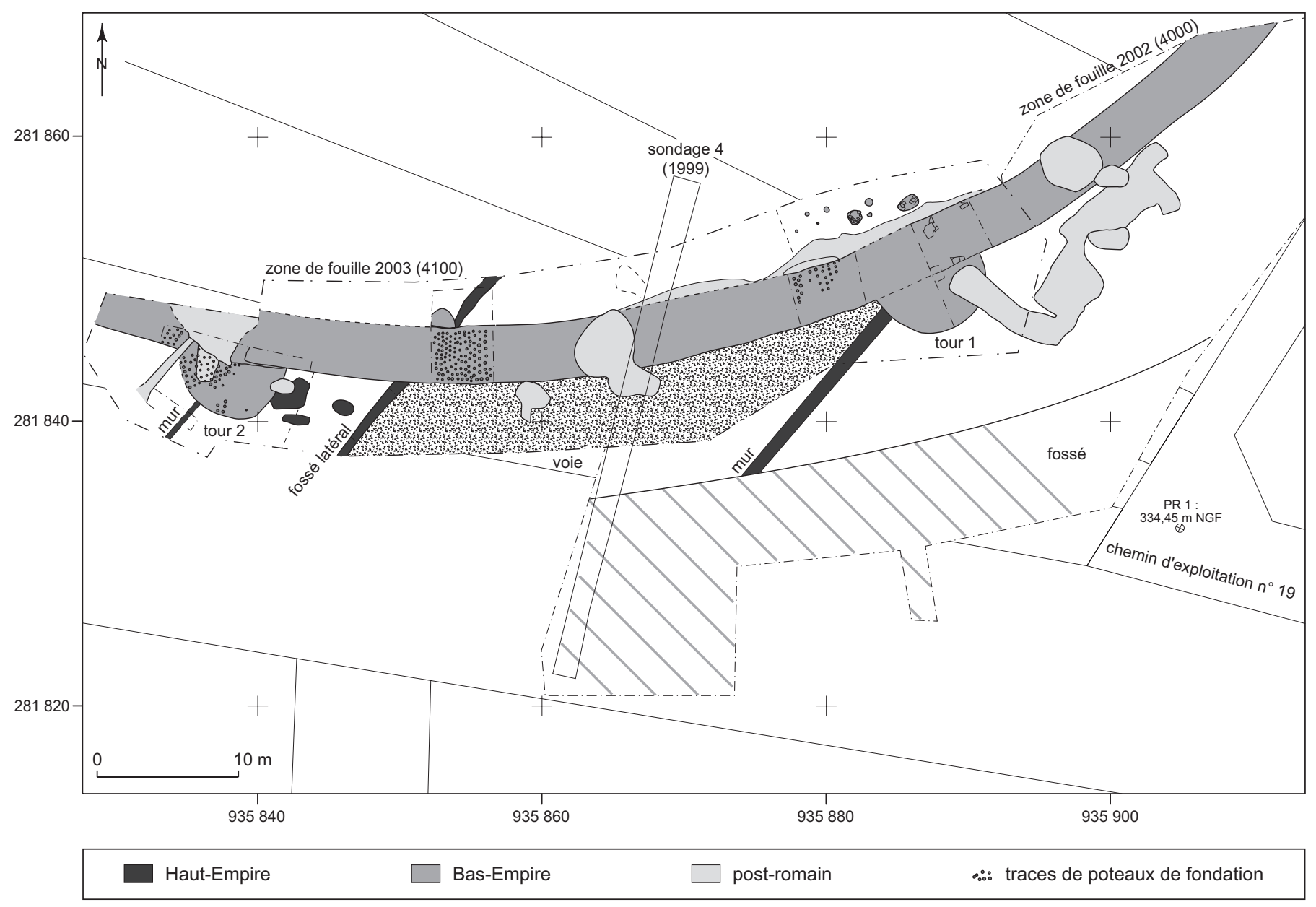

Fig. 29 - Plan d'ensemble des fouilles menées sur la fortification de Mandeure en 2002 et 2003 (DAO : G. Kuhnle, INRAP).

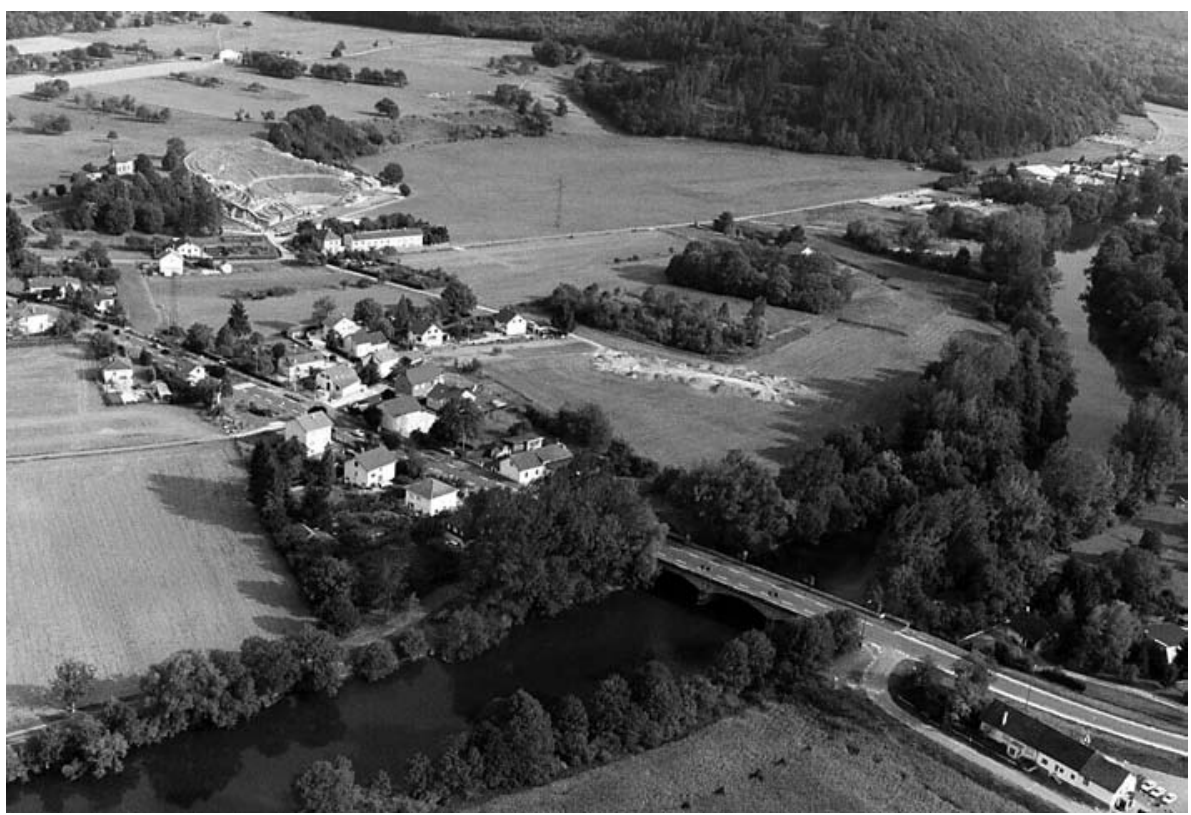

Fig. 30 - Vue aérienne du théâtre, du sanctuaire et de la fortification de Mandeure, situés sur la rive droite du Doubs ; la zone de fouille de 2003 sur la partie sud de l'enceinte est visible (cliché : G. Kuhnle, INRAP). 


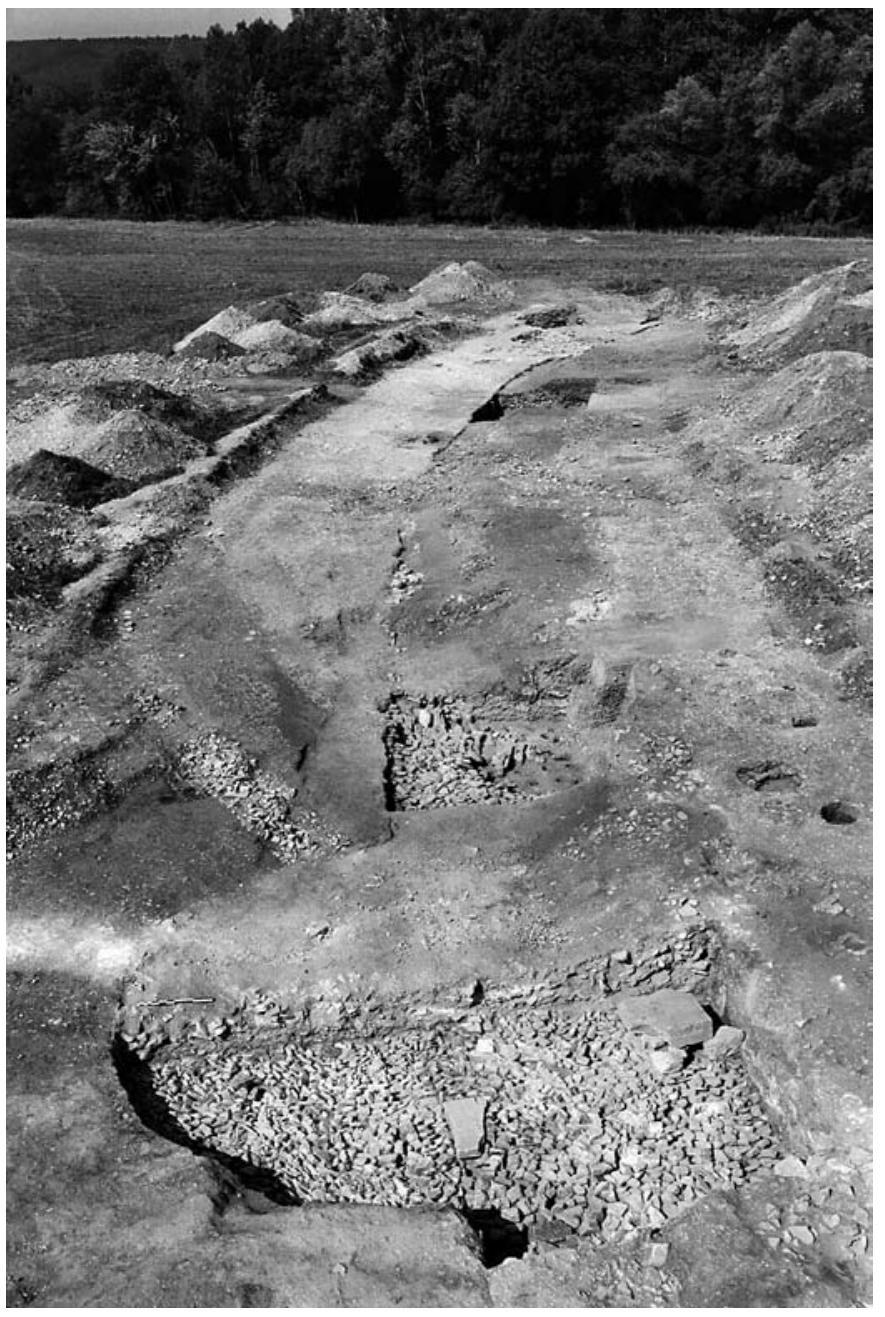

Fig. 31 - Vue du nord-est du mur d'enceinte de Mandeure avec la tour 1 en premier plan; on note quelques grands blocs posés à plat sur le hérisson de pierres (cliché : G. Kuhnle, INRAP).

ouvert entre les tours 1 et 2 (fig. 33). On compte 110 trous de piquet sur une surface d'à peine $16 \mathrm{~m}^{2}$, soit en moyenne presque 7 piquets $(6,875$ exactement) par mètre carré. En réalité, on peut trouver jusqu'à 11 piquets par mètre carré. Ils sont espacés de $0,05 \mathrm{~m}$ à $0,40 \mathrm{~m}$, soit en moyenne d'une vingtaine de centimètres. Au niveau du fond de la tranchée, ces trous de piquet ont un diamètre qui oscille entre $0,10 \mathrm{~m}$ et $0,22 \mathrm{~m}$; à $0,20 \mathrm{~m}$ sous ce niveau, leur diamètre ne mesure plus qu'entre $0,10 \mathrm{~m}$ et $0,14 \mathrm{~m}$. Nous n'avons jamais atteint le fond de ces trous car les piquets étaient sans aucun doute profondément enfoncés dans le substrat graveleux. Pour comparaison, les rondins de chêne épointés de faible diamètre, employés dans le pilotis des murs extérieurs de la fortification tardive d'Aegerten-Isel (Suisse) avaient une longueur moyenne de $3 \mathrm{~m}$ (Bacher, Suter, 1989).

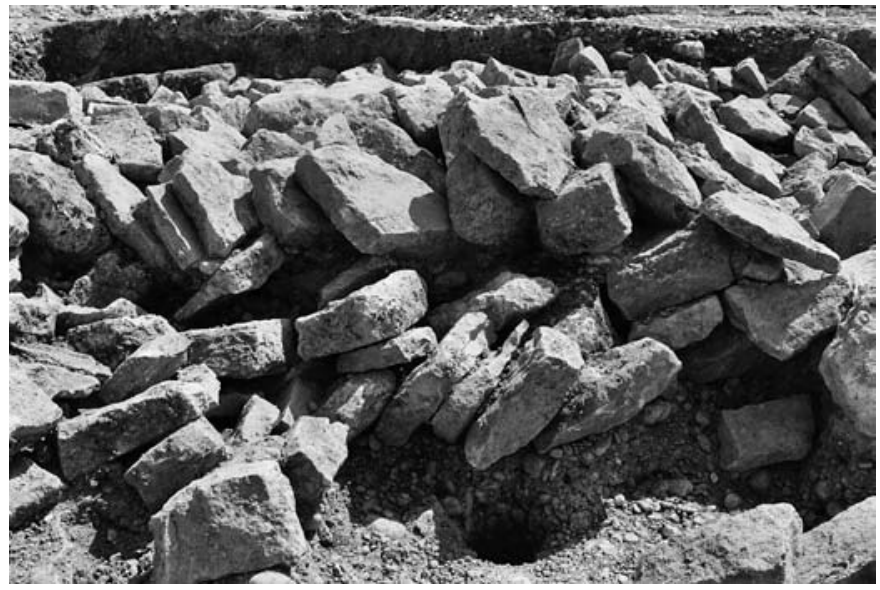

Fig. 32 - Vue du nord-ouest du détail des deux niveaux de hérisson conservés dans la fondation du mur d'enceinte de Mandeure à l'ouest de la tour 1 (cliché : G. Kuhnle, INRAP).

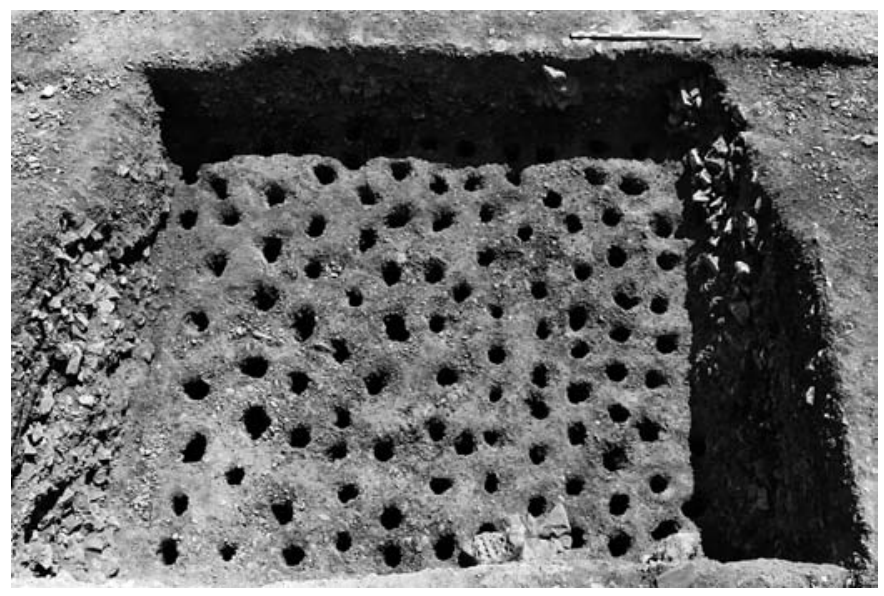

Fig. 33 - Radier de pieux battus au fond de la tranchée de fondation du mur d'enceinte de Mandeure entre les tours 1 et 2 (vue du nord) (cliché : G. Kuhnle, INRAP).

La fine couche grisâtre, composée d'un mélange d'argile, de sable et de gravier, qui couvre par endroits le fond de la tranchée peut être mise en relation avec la présence d'une équipe d'ouvriers dans la tranchée lors de l'enfoncement des piquets et de la mise en place de la première strate de pierres. Certains piquets n'avaient pas été entièrement enfoncés puisque leurs têtes dépassaient cette couche de travail, comme en témoignent les vides observés dans la première strate de pierres, à l'aplomb des trous de piquet.

La semelle de fondation du mur d'enceinte est réalisée en pierres sèches. Les pierres, dont de nombreux spolia, sont posées en biais de façon à constituer un hérisson, 
orienté dans le sens du mur (fig. 32). Le toit de chaque strate est approximativement nivelé à l'aide d'une couche de limon légèrement argileux, bien tassée, qui facilite la mise en place des pierres du niveau suivant. Les pierres sont alors disposées dans le sens opposé à celui de la strate sous-jacente. Au mieux, trois niveaux du hérisson sont conservés.

Dans la tranchée de la courtine, à l'arrière de la tour 1 , quelques grands blocs posés à plat constituent les restes de deux assises surmontant le hérisson (fig. 31). L'assise inférieure, haute de $0,20 \mathrm{~m}$ à $0,28 \mathrm{~m}$, est constituée de blocs de taille différente qui sont liés à du limon argileux brun incluant du gravier et des cailloux. De l'assise supérieure, il ne reste qu'un seul bloc de grande taille mesurant $0,87 \mathrm{~m} \mathrm{x}$ 0,82 m x 0,28 m. Cet aménagement témoigne certainement de la technique de fondation employée de façon systématique au-dessus de la semelle de fondation constituée par le hérisson de pierres. S'il reste si peu d'éléments en place, c'est que ces grands blocs étaient plus intéressants pour les spoliateurs que les pierres de moindre calibre ou les éléments sculptés.

De manière générale, la largeur de la fondation (plus de $3 \mathrm{~m}$ ), la présence de tours saillantes vers l'extérieur et l'emploi de spolia sont typiques des enceintes du Bas-Empire. Les indices de datation liés à l'existence du radier de pilotis offrent une fourchette trop vaste puisque des pieux ou piquets de chêne employés en fondation se rencontrent au Haut- et au Bas-Empire, surtout dans des secteurs marécageux ou instables. Certes, le pilotis se retrouve couramment sous les fondations des enceintes du Bas-Empire, peut-être aussi parce que celles-ci sont en général beaucoup plus larges que celles du Haut-Empire. En revanche, on note à Mandeure l'absence d'un radier de poutres, aménagé sous ou/et dans la semelle de fondation, en relation avec le radier de pieux ${ }^{21}$.

G. K.

21. L'emploi de poutres semble caractéristique - en l'état actuel de la recherche dans la partie de l'Empire romain concernée (soit surtout en Allemagne et en Suisse) - des fortifications érigées sous le règne de Valentinien. Le dernier exemple mis au jour, cette fois-ci situé en Alsace, est la fortification d'Altkirch à Biesheim-Oedenbourg, fouillée par l'équipe du professeur Nuber de Fribourg dans le cadre du projet franco-allemand-suisse (direction: M. Reddé) (Nuber, Reddé, 2002, p. 225-226). La technique de construction observée à Biesheim et celle employée dans les fondations de la tour défensive du lieu-dit Kleiner Laufen/Summa Rapida (Suisse) au bord du Rhin, datée par une inscription de Valentinien, sont identiques (Stehlin, von Gonzenbach, 1957, p. 114 ; CIL, XIII, 11537).

\section{SYNTHÈSE SUR LA TOPOGRAPHIE ET L'ARCHITECTURE DU SITE FORTIFIÉ AU BAS-EMPIRE}

La superposition et la mise en corrélation de toutes les données topographiques disponibles à ce jour permettent de faire un certain nombre d'observations sur le site fortifié au Bas-Empire.

Le tracé du fossé défensif est connu sur presque toute sa longueur si l'on juxtapose la carte magnétique, sur laquelle on peut le suivre sur plus d'une centaine de mètres, et le cadastre napoléonien sur lequel les parcelles affectent un arc de cercle (pl. XIII, hors texte). Le fossé a la forme d'une cloche dont la base est constituée par le Doubs (fig. 30).

Délimité par la berge du Doubs et le fossé, le terrain plat pouvant servir d'assise à la fortification maçonnée mesure jusqu'à $170 \mathrm{~m}$ dans le sens parallèle au cours d'eau (nord-nord-est/sud-sud-ouest) et jusqu'à $140 \mathrm{~m}$ dans le sens perpendiculaire (ouest-nord-ouest/est-sud-est). Les murgers repérés par F. Morel-Macler sur le site de la fortification sont tous localisés sur cette «plate-forme» (pl. XIV, hors texte).

Le front nord-nord-est de la fortification se trouve de toute évidence dans le secteur de la rue du Pont. Si on se fie à la forme du parcellaire du cadastre napoléonien et au grand murger (A) dessiné par Morel-Macler, l'enceinte passerait sous les maisons actuelles aux $\mathrm{n}^{\text {os }} 136,138$ et 140 de la rue du Pont (pl. XIII et XIV, hors texte). En effet, on peut supposer que les trois parcelles incurvées correspondent au fossé et le grand murger (A) à l'enceinte.

$\mathrm{Au}$ sud de la rue du Pont, nos connaissances sur les fronts de la fortification sont mieux renseignées, grâce essentiellement à la portion du front sud-sud-est qui a pu être étudiée sur $85 \mathrm{~m}$ de long en aire ouverte lors des deux campagnes de 2002 et 2003 ; d'une largeur supérieure à 3 m en fondation, elle est incurvée et munie de deux tours semicirculaires, distantes de $50 \mathrm{~m}$ d'axe en axe.

À la lumière des données de fouille, le segment muni de la tour 1 est finalement bien lisible sur la carte de prospection magnétique (pl. XIII, hors texte). En revanche, le secteur du front sud avec la tour 2 l'est moins bien, mais son prolongement vers le Doubs y est à nouveau perceptible. Deux petits murgers $(\mathrm{F}, \mathrm{G})$ et le point 11 du relevé de $\mathrm{F}$. Morel-Macler se trouvent localisés sur son tracé (rappelons que le point 11 est le lieu de découverte des spolia attribuées par l'auteur au temple de Jupiter Ammon) (pl. XIV, hors texte). Un troisième petit murger $(\mathrm{H})$ pourrait marquer l'angle de l'enceinte si la berge antique correspondait à peu près à la berge actuelle. Enfin, le sondage B du gazoduc se situe aussi dans 
le prolongement du front sud, en bas de la berge. Y. Jeannin y a observé les restes d'un mur ou d'un massif maçonné qu'il interprète comme une digue et qui pourrait rejoindre le Doubs à la manière des enceintes des burgi avec embarcadère (Heukemes, 1981), à moins qu'il faille y voir un aménagement de berge plus « classique ».

En ce qui concerne le front nord-ouest de la fortification, plusieurs données convergentes nous renseignent sur l'hypothétique tracé de l'enceinte parallèle au Doubs.

La zone 3000 (fouille programmée 1999) a livré une tranchée de fondation perturbée par des creusements postromains et mesurant environ 2,70 $\mathrm{m}$ de large (pl. XII, hors texte). Grâce aux fouilles extensives menées dans le cadre du PCR (2002 et 2003), les vestiges lacunaires d'un radier de pilotis et d'une semelle de fondation en pierres sèches posées en hérisson dans la zone 3000 peuvent être interprétés comme typiques du système fortifié de Mandeure.

Il est intéressant de constater que l'emplacement et l'orientation nord-nord-est/sud-sud-ouest de ce petit tronçon d'enceinte concordent avec la courbe des 135 m NGF dont la signification a déjà été remarquée (pl. XIV, hors texte). De plus, on distingue sur la carte de la prospection magnétique une anomalie sombre et rectiligne dont l'axe n'est décalé que de très peu par rapport à celui du tronçon d'enceinte observé en zone 3000 (pl. XII et XIII, hors texte). Si l'on prolonge de manière rectiligne le tracé de l'anomalie magnétique, celui-ci rejoint au sud-sud-ouest le petit murger d'angle $(\mathrm{H})$ et au nord-nord-est l'extrémité nord-ouest du grand murger (A). La concordance entre la microtopographie, l'anomalie magnétique, la localisation des murgers et la découverte de la tranchée de fondation dans la zone 3000 suggère ainsi un tracé probable du front nord-ouest de la fortification (fig. 40 et pl. XIV, hors texte).

Dans la mesure où la zone 3000 se situe près de l'axe de la fortification perpendiculaire au Doubs, on ne peut toutefois exclure que la fondation coupée appartienne déjà à une hypothétique porte d'accès aux berges aménagées en port.

Excepté cette hypothétique porte ou tour-porte et d'éventuelles tours d'angle qui pouvaient être saillantes vers le Doubs, nous supposons l'absence de tours du côté du cours d'eau. On serait donc en présence d'un mur d'enceinte rectiligne, dépourvu de tours intermédiaires, dont l'épaisseur semble être plus faible par rapport à celle de l'enceinte incurvée et munie de tours intermédiaires. Ce double phénomène - mur moins épais et absence de tours le long d'une voie d'eau - a été observé sur plusieurs fortifications du Bas-Empire appuyées à un cours d'eau (Kuhnle, 1991, p. 71-82).
En ce qui concerne l'axe nord-ouest/sud-est de la fortification, il est assuré à la fois par la prospection magnétique (pl. XIII, hors texte) et par la présence de trois murgers (B, C et D) disposés en enfilade (pl. XIV, hors texte). Il est important de noter que le sondage A du gazoduc (existence possible d'une pile de pont) se situe dans le prolongement de cet axe nord-ouest/sud-est. En revanche, le point 12 intitulé "Culée d'un pont» sur la carte topographique de F. MorelMacler (1847) et la structure interprétée comme étant une «Ancienne culée d'un pont» sur le document de 1893 sont tous deux beaucoup trop décalés par rapport à cet axe. S'il y a eu pont, il doit se trouver entre ces deux emplacements cartographiés et passer approximativement par l'endroit où est localisé le sondage A du gazoduc. Nous émettons donc l'hypothèse que l'axe nord-ouest/sud-est corresponde à une rue bordée de bâtiments qui sort de l'enceinte au nord-ouest par une porte pour accéder au port et qui pourrait aussi être prolongée par un pont traversant le Doubs.

Si cette hypothèse devait se confirmer, une question importante se poserait pour le front sud-est de la fortification où la prospection magnétique n'a pas pu être effectuée faute de possibilité d'accès. En effet, un petit murger (E) double celui de l'axe médian qui se trouve le plus au sud-est (D) et les courbes du levé microtopographique forment au même endroit (au nord-est de la zone 2700) une excroissance par rapport au tracé supposé du mur d'enceinte (pl. XIII et XIV, hors texte). Il pourrait alors s'agir d'une tour, voire d'une porte-tour ${ }^{22}$.

Enfin, à l'extérieur du fossé, F. Morel-Macler a relevé deux murgers (I et J) qui se trouvent dans le prolongement de l'axe perpendiculaire au Doubs ; malheureusement inaccessibles car localisés sous quatre constructions modernes (n ${ }^{\text {os }}$ 1bis-3-5, impasse du Clos), ils couvrent une surface de $48 \mathrm{~m}$ sur $36 \mathrm{~m}$. Ce n'est probablement pas un hasard si plusieurs monnaies du $\mathrm{IV}^{\mathrm{e}} \mathrm{s}$. et les fragments de la statue colossale de Mars proviennent de cet endroit (Pichot, 1995a et b). La découverte en 2003, dans la tranchée d'épierrement de l'enceinte, d'un avant-bras de statue en marbre qui recolle presque Bruch am Bruch avec la main de l'effigie colossale de Mars, découverte un siècle plus tôt, pourrait d'ailleurs confirmer l'appartenance de ces murgers à la fortification. On pourrait alors imaginer la présence de la tête d'un pont permettant d'enjamber le fossé et d'accéder par l'hypothétique porte à la fortification.

G. K.

22. Les fouilles programmées en 2006-2007, dirigées par J. Monnier (université de Lausanne), porteront sur cette zone. 
Tabl. I - Répartition des monnaies du IV s. par période et par atelier.

\begin{tabular}{|c|c|c|c|c|c|c|c|c|c|c|c|c|c|c|c|c|c|}
\hline & Londres & Trèves & Lyon & Arles & Ticinum & Rome & Aquilée & Scicia & Thessalonique & Heraclea & Constantinople & Nicomédie & Cyzique & Antioche & Imitations & Ind. & Total \\
\hline 294-307 & - & - & 1 & - & 1 & - & - & - & - & - & - & - & - & - & - & - & 2 \\
\hline $307-318$ & 1 & 4 & 1 & 1 & - & - & - & - & - & - & - & - & - & - & - & - & 7 \\
\hline $318-330$ & 7 & 15 & 5 & 4 & 4 & - & - & 2 & 1 & - & - & - & - & - & - & 6 & 44 \\
\hline $330-341$ & - & 33 & 23 & 16 & - & 10 & - & 3 & 1 & 3 & 2 & 1 & 1 & 2 & 26 & 35 & 156 \\
\hline $341-348$ & - & 16 & 11 & 4 & - & 1 & - & - & - & - & - & - & - & - & 7 & 16 & 55 \\
\hline $348-353$ & - & 3 & 6 & - & - & - & - & - & - & - & - & - & - & - & 2 & - & 11 \\
\hline $353-364$ & - & - & - & 1 & - & - & - & - & - & - & - & - & - & - & 1 & 1 & 3 \\
\hline $364-378$ & - & 1 & 21 & 14 & - & 4 & 2 & 4 & - & - & - & - & - & - & - & 12 & 58 \\
\hline ind. IV $\mathrm{s}$. & - & - & - & - & - & - & - & - & - & - & - & - & - & - & - & 20 & 20 \\
\hline Total & 8 & 72 & 68 & 40 & 5 & 15 & 2 & 9 & 2 & 3 & 2 & 1 & 1 & 2 & 36 & 90 & 356 \\
\hline
\end{tabular}

\section{LES MONNAIES}

Les opérations archéologiques réalisées au lieu-dit Champs des Cloux du Château jusqu'en 2004 ont livré 364 monnaies antiques.

Le premier lot est composé de 43 monnaies découvertes avant 1998 sur le site de la fortification, essentiellement lors de la construction de maisons individuelles (liste aimablement fournie par Y. Jeannin) et au cours de prospections réalisées par MM. Augé, Chambrelland, Lame, Mazimann et Mougin. Ce lot a déjà fait l'objet d'une étude préliminaire dans la synthèse consacrée à Mandeure au $\mathrm{IV}^{\mathrm{e}} \mathrm{s}$. (Mazimann, 1992).

Le deuxième lot, fort de 266 monnaies, est issu des fouilles menées en 1998 et 1999. Ces monnaies proviennent de l'opération Behra (fouille Mazimann et Mougin, 1998), de la tranchée de la parcelle 7 (zones 2200 à 2700 : fouille Mazimann, 1998) et de son prolongement vers l'ouest (zone 3000 : fouille Mougin, 1999), ainsi que des prospections au détecteur de métaux, réalisées sur les déblais de ces interventions par MM. Guillot, Grut et Mottaz.

Le troisième lot se compose des 55 monnaies trouvées pendant les deux campagnes de fouilles programmées du PCR (fouilles Kuhnle, 2002 et 2003).

Il est à noter que les monnaies retrouvées en fouille in situ sont rares et ne constituent pas plus de $13 \%$ de l'ensemble, ce qui n'empêche en rien l'étude de la circulation monétaire.

Les 364 monnaies découvertes sur la fortification tardive peuvent être classées en quatre groupes chronologiques.

Le premier concerne un potin gaulois, non attribuable.

Le deuxième est constitué de trois monnaies dont un as au nom de Tibère frappé à Lyon entre 9 et 14 apr. J.-C., un autre probablement au nom de Claude frappé à Rome entre
41 et 54 et une imitation non déterminée frappée au $\mathrm{I}^{\mathrm{er}} \mathrm{s}$. ou au $\mathrm{II}^{\mathrm{e}} \mathrm{s}$.

Le troisième regroupe quatre antoniniens : un au nom de Gallien frappé entre 260 et 268, un autre au nom de Victorin frappé entre 268 et 270 et deux imitations, dont une au nom de Tetricus, frappées dans le troisième quart du III $^{\mathrm{e}} \mathrm{s}$.

Le quatrième groupe, le plus important, se compose de 356 monnaies frappées entre 299 et 378 . Il peut être divisé en sous-groupes reprenant les périodes chronologiques établies par R. Reece (1987) (tabl. I).

\section{PÉRIODE ALLANT DE 294 (RÉfORME MONÉTAIRE DE DIOCLÉTIEN) À 318}

Elle est représentée par 9 monnaies frappées dans les ateliers de Londres (1 ex.), Trèves (4 ex.), Lyon (2 ex.), Arles (1 ex.) et Ticinum (1 ex.). Deux monnaies datent de la réforme de 294 : un nummus au nom de Maximien (Hercule ou Galère) et un antoninien de Dioclétien. Les autres monnaies ont été frappées après la troisième dévaluation du nummus en 310 et sont toutes au nom de Constantin. Les types présents sur les 9 revers sont Soli invicto comiti (6 ex.), Genio populi romani (1 ex.), Marti conserv (1 ex.) et une marque votive VOT/XX (1 ex.).

\section{PÉRIODE ALLANT DE 318 À 330}

Elle compte 44 nummi issus des ateliers de Londres (7 ex.), Trèves (15 ex.), Lyon (5 ex.), Arles (4 ex.), Ticinum (4 ex.), Siscia ( 2 ex.) et Thessalonique (1 ex.) ; les marques de provenance des 6 nummi restants ne sont pas identifiables. Les droits sont majoritairement au nom de Constantin (26 ex.), puis de ses fils : Constantin II (5 ex.) et Crispus 
(7 ex.). Les autres monnaies représentent Licinius (1 ex.) et son fils Licinius II (3 ex.). Les droits de deux pièces de cette période ne sont pas identifiés. Les revers sont plus diversifiés avec les types suivants : Soli invicto comiti (2 ex.), Iovi conservatori (1 ex.), Victoria caess nn (1 ex.), Victoriae laetae princ perp (9 ex.), Dominorum nostrorum caess (2 ex.), Virtus exercit (2 ex.), Beata tranquillitas (11 ex.), Caesarum nostrorum (2 ex.), D $n$ constantini max aug (1 ex.), Sarmatia devicta (6 ex.), Providentiae augg (4 ex.), Virtus augg (1 ex.) ; deux sont indéterminés.

\section{PÉRIODE ALLANT DE 330 À 348}

Forte de 211 nummi, elle est la plus abondamment représentée sur le site. Les ateliers présents sont Trèves (49 ex.), Lyon (34 ex.), Arles (20 ex.), Rome (11 ex.), Siscia (3 ex.), Thessalonique (1 ex.), Heraclée (3 ex.), Constantinople (2 ex.), Nicomédie (1 ex.), Cyzique (1 ex.) et Antioche (2 ex.). L'origine de 51 monnaies n’a pu être déterminée du fait de leur mauvais état de conservation. 33 monnaies sont de provenance «locale» puisque ce sont des imitations aux noms de Constantin (1 ex.), Constantin II (1 ex.), Constance II (8 ex.), Constant (2 ex.), Rome (3 ex.), Constantinople ( 4 ex.), Constance II ou Constant ( 5 ex.) et 9 imitations indéterminées.

Les frappes officielles sont aux noms de Constantin (21 ex.), Constantin II (19 ex.), Constance II (19 ex.), Constant (16 ex.), Delmace (1 ex.), Hélène (4 ex.), Théodora (1 ex.), Rome (17 ex.), Constantinople (18 ex.) et 14 sont indéterminées.

Les revers de cette période forment deux groupes. Le premier concerne les types frappés sous le règne de Constantin : Gloria exercitus à deux enseignes (40 ex.), la Louve et les Jumeaux (20 ex.), la Victoire sur une proue (22 ex.) et Gloria exercitus à une enseigne (57 ex.). Le second rassemble les types frappés sous le règne des fils de Constantin: Aeterna pietas (2 ex.), Constantin sur un quadrige (7 ex.), Pax publica (4 ex.), Pietas romana (1 ex.), Virtus augg nn (1 ex.), VN-MR (1 ex.) et Victoriae dd augg $q$ nn (54 ex.). Seuls deux revers sont indéterminés.

\section{PÉRIOdE ALLANT DE 348 À 364}

Elle compte 14 monnaies dont 11 maiorinae (aes II), 2 maiorinae légères (aes III) et 1 demi-maiorina (aes IV). Neuf monnaies proviennent des ateliers de Trèves (3 ex.) et de Lyon (6 ex.) ; la provenance de deux autres est «locale " puisqu'il s'agit d'imitations au nom de Magnence alors que celle des trois monnaies restantes est indéterminée. Les frappes officielles présentent les portraits de Constance II (2 ex.), Constant (2 ex.), Magnence (7 ex.) et Julien, César (1 ex.). Les revers portent les types suivants : Fel temp reparatio (5 ex.), Gloria romanorum (5 ex.), Victoriae dd nn aug et cae (1 ex.), Felicitas rei publice (2 ex.) et Spes rei publice (1 ex.).

\section{PÉRIODE ALLANT DE 364 À 378}

Cinquante-huit monnaies concernent l'ère valentinienne. Ce sont des maiorinae légères qui ont été frappées dans les ateliers de Trèves (1 ex.), Lyon (21 ex.), Arles (14 ex.), Rome (4 ex.), Aquilée (2 ex.) et Siscia (4 ex.), 12 exemplaires n'étant pas attribuables ; 12 droits sont dédiés à Valentinien, 23 à Valens et 15 à Gratien, 8 restent indéterminés. Les revers se répartissent entre trois types: Gloria romanorum (22 ex.), Securitas rei publicae (31 ex.) et Gloria novi saeculi (5 ex.).

Enfin, au groupe des monnaies du $\mathrm{IV}^{\mathrm{e}} \mathrm{s}$. s'ajoutent 20 monnaies indéterminées, attribuables par leur aspect à ce siècle.

\section{SYNTHÈSE}

Les monnaies romaines découvertes dans l'emprise de la fortification du Bas-Empire couvrent un arc chronologique compris entre 9 et 378 apr. J.-C.

$\mathrm{Au}$ sein de cette plage temporelle, il est possible de dégager deux ensembles cohérents.

Le premier concerne les trois monnaies frappées avant 260. Celles-ci témoignent de l'occupation antérieure à l'édification de la fortification (niveaux non fouillés). Les deux frappes officielles proviennent l'une de Rome et l'autre de Lyon.

Le second ensemble comprend les monnaies frappées entre 260 et 378 . Les quatre antoniniens sont à rapprocher des 356 monnaies du IV ${ }^{\mathrm{e}}$ s., puisqu'ils peuvent être en circulation jusque dans les années 300. Les ateliers de provenance de ces monnaies sont nombreux puisque, depuis la réforme de Dioclétien en 294, la frappe du numéraire est décentralisée. En outre, la situation géographique de Mandeure sur un axe de circulation important explique la présence de tous ces ateliers, même si les officines les mieux représentées sont les plus proches du site: Trèves avec 72 ex., Lyon avec 68 ex. et Arles avec 40 ex.

La répartition des monnaies du $\mathrm{IV}^{\mathrm{e}} \mathrm{s}$. se caractérise par deux temps forts, l'un à l'époque constantinienne et l'autre à l'époque valentinienne (fig. 34). 


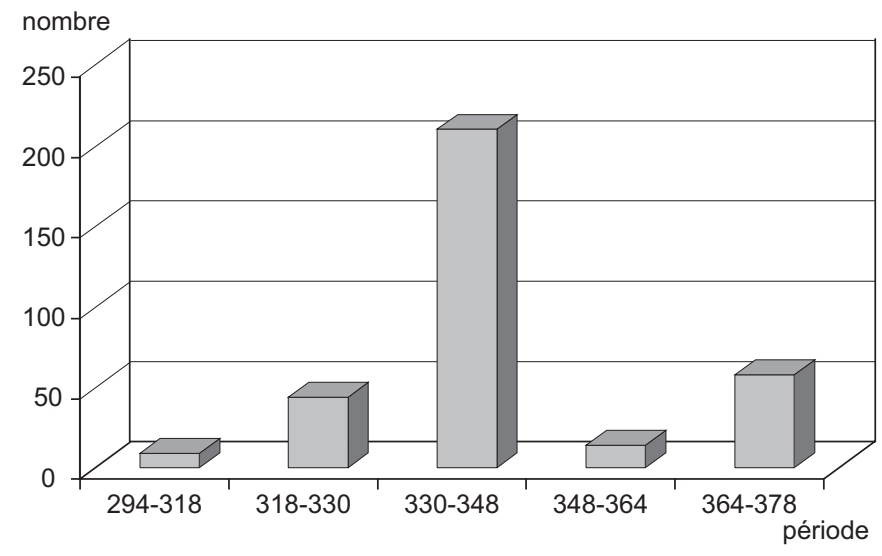

Fig. 34 - Histogramme de répartition des monnaies selon les périodes définies par R. Reece (d'après Reece, 1987).

Le premier est marqué par les 211 nummi de la période 330-348. Cette forte représentation nous conduit à penser que c'est durant cette période que la fortification est édifiée par les militaires, seuls capables de concevoir et construire un tel ouvrage.

La faible représentation des monnaies de la période suivante (14 monnaies frappées entre 348 et 364) correspond à un phénomène déjà connu dans la région. Les problèmes d'approvisionnement en numéraire frais ne doivent pas forcément être mis en relation avec un abandon du site, mais peuvent plus vraisemblablement s'expliquer par les troubles politiques de la période comme l'usurpation de Magnence et «l'invasion de 352 ». Ces événements contribuent à ralentir le commerce et la circulation monétaire. Cette dernière, fortement affaiblie, devait avant tout être liée aux déplacements de contingents militaires.

Le nombre de monnaies de la période 364-378 (58 ex.), largement plus important que celui de la période précédente, peut coïncider avec une (ré)occupation militaire de la fortification.

L'abandon de la fortification semble être intervenu dès la fin de l'époque valentinienne puisqu'aucune monnaie de la période suivante (378-402) n'a été mise au jour sur la fortification.

A. B., J.-P. M.

\section{LES PETITS OBJETS}

Les différentes opérations archéologiques menées sur la fortification tardive de Mandeure ont permis de mettre au jour 27 objets rattachés à cette période. Ces objets peuvent être classés en deux groupes fonctionnels: les militaria (9 ex.) auxquels s'ajoutent les fibules cruciformes (8 ex.), considérées comme liées à la sphère militaire puisqu'elles étaient portées par les soldats et par les dignitaires de l'Empire, et la parure féminine (10 ex.).

Parmi les neuf militaria identifiés, on compte :

- 1 fer de lance en forme de feuille de saule et à épaulement bas, typologiquement mal daté (fig. 35, $\mathrm{n}^{\circ} 1$ ) ;

- 1 lest de plumbata dont l'identification est presque certaine (fig. 35, no 2). L'apparition de ce type d'objet est datée du IV $^{\mathrm{e}}$ s. (Bishop, Coulston, 1993 ; Feugère, 1993) ;

- 1 ou 2 pontets de suspension de fourreau de spatha (fig. 35, $\mathrm{n}^{\text {os }} 3$ et 4), l'épée longue dévolue aux fantassins aux III $^{\mathrm{e}}$ et $I^{e} s$. L'exemplaire $n^{\circ} 3$, bien identifié, est atypique. Il peut être rapproché du type Carnap-Bornheim IIIC dont il pourrait constituer une variante inédite (Carnap-Bornheim, 1991). L'identification du fragment $n^{\circ} 4$ est plus incertaine ; - 1 plaque-boucle de ceinturon apparentée à la classe 1, forme A de Sommer (Sommer, 1984) et datée de la première moitié du IV ${ }^{\mathrm{e}}$ s. (fig. 35, $\mathrm{n}^{\mathrm{o}} 5$ ) ;

- 1 ferret de ceinturon proche des modèles attestés au IV $\mathbf{s}$. (fig. 35, no 6) (Aurrecoechea-Fernandez, 2001) ;

- 1 plaque décorative de ceinturon ornée d'ocelles estampées et de lignes parallèles incisées, caractéristiques $\mathrm{du} \mathrm{IV}^{\mathrm{e}} \mathrm{s}$. (fig. 35, no 7) (Boube-Picot, 1994) ;

- 2 appliques de harnachement, appartenant à un type tant militaire que civil, en usage du $\mathrm{II}^{\mathrm{e}}$ au $\mathrm{IV}^{\mathrm{e}}$ s. (fig. 35, $\mathrm{n}^{\mathrm{os}} 8$ et 9) (Voirol, 2000).

Sur les huit fibules cruciformes mises au jour, trois appartiennent au type Keller-Pröttel 1 (fig. 36, $\mathrm{n}^{\text {os }} 1-3$ ). Les fibules $\mathrm{n}^{\text {os }} 1$ et 2 sont rattachées à la variante $\mathrm{A}$ alors que la $n^{\circ} 3$ est apparentée à la variante B. Elles sont toutes trois datées typologiquement des années 280-320 (Pröttel, 1988, p. 352). Les fibules $\mathrm{n}^{\text {os }} 4-6$ et 8 de la figure 36 appartiennent au type Pröttel 3/4, variante $\mathrm{B}$ pour les $\mathrm{n}^{\mathrm{os}} 4$ à 6 , variante $D$ pour le $n^{\circ} 8$. D'autre part, il est fort possible que le bouton $\mathrm{n}^{\mathrm{0}} 7$ appartienne également à une fibule de ce type. Les fibules de type Pröttel 3/4 sont datées des années 330-340 à 410 (Pröttel, 1988, p. 363).

Au sein des dix objets de parure féminine mis au jour (fig. 37), on dénombre quatre perles en verre (fig. 37, $\mathrm{n}^{\text {os }} 1$ à 4), dont les types sont tous attestés dans les contextes tardifs (Riha, 1990 ; Swift, 2000).

Parmi les quatre bracelets découverts, deux (fig. 37, $n^{\text {os }} 7-8$ ) sont en métal (alliage cuivreux), un en verre ( $\left.n^{\circ} 9\right)$ et le dernier en schiste $\left(n^{\circ} 10\right)$. Ils se rattachent tous à des types tardifs (Riha, 1990 ; Swift, 2000).

Deux épingles à cheveux ont également été découvertes (fig. $37, \mathrm{n}^{\text {os }} 5$ et 6 ). Typologiquement mal datées, elles sont, 


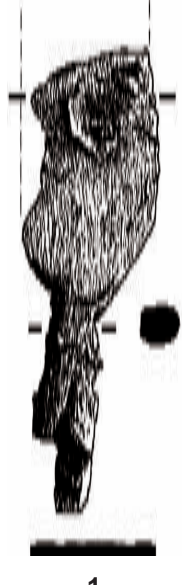

1

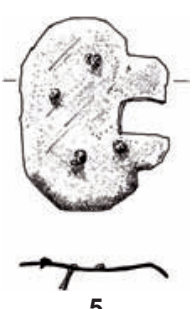

5
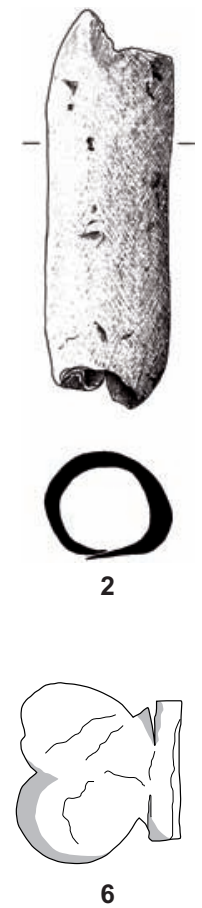
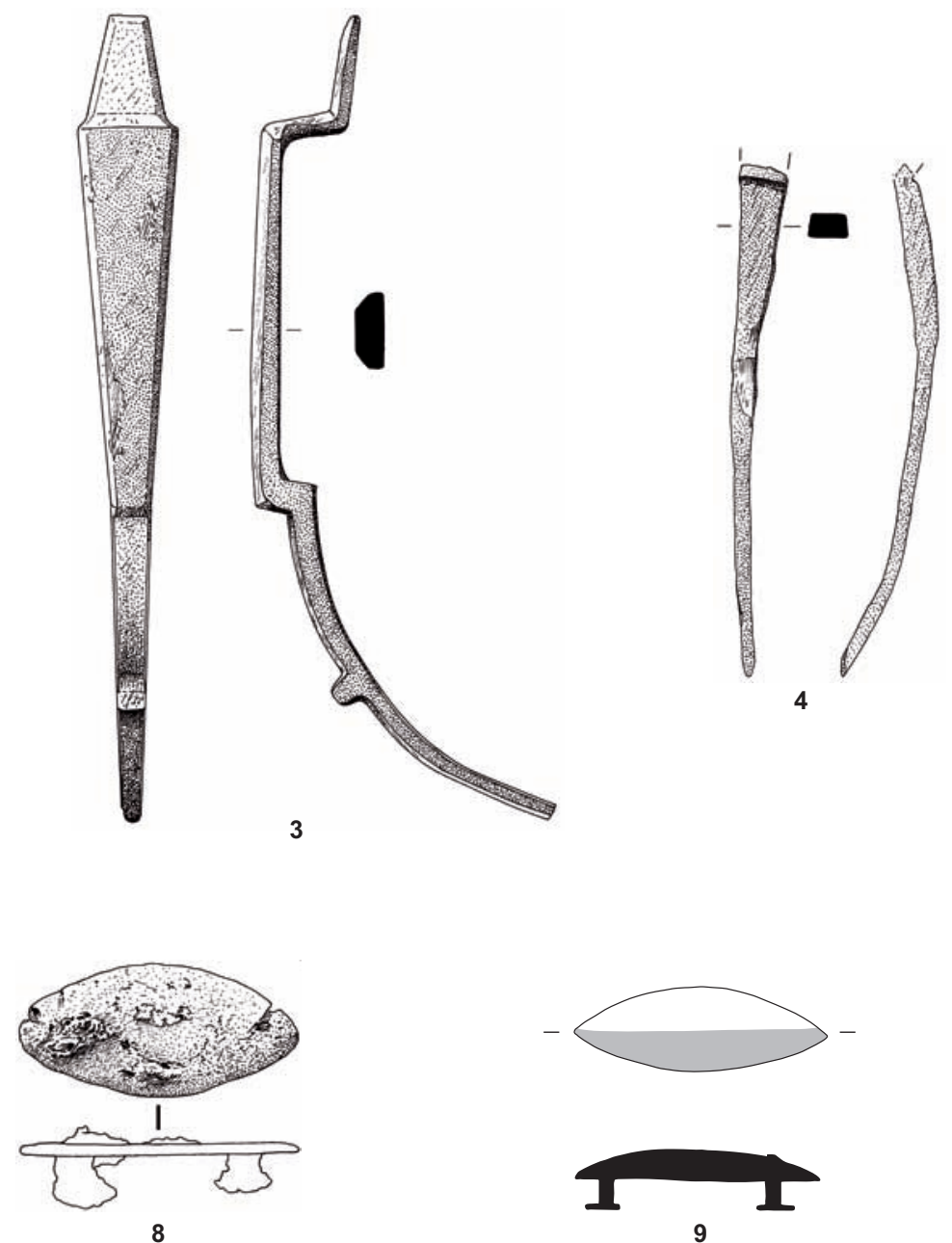

0 $5 \mathrm{~cm}$

Fig. 35 - Militaria provenant de Mandeure : 1, fer de lance; 2, lest de plumbata ; 3-9, équipement en alliage cuivreux (dessins : $n^{o s}$ 1-3, 5, 7, 8, J. Gelot, INRAP; $n^{o}$ 4, I. Dard ; nos 6, 9, J.-C. Mottaz, ARESAC).

de par leur contexte de découverte, liées à l'occupation de la fortification.

La partition des petits objets du Bas-Empire découverts sur le site de la fortification de Mandeure en deux lots seulement, celui des militaria et celui de la parure, nous indique à la fois une fréquentation du site par des militaires, comme on le supposait a priori pour ce type de fortification, et une fréquentation par des civils comme le suggèrent les objets de parure féminine.

Les petits objets n'étant pas de très bons fossiles directeurs, ils ne nous permettent pas d'affiner la chronologie du site. Toutefois, les fibules cruciformes confortent les données chronologiques livrées par les monnaies. Elles forment deux lots : celui des fibules de type Keller-Pröttel 1, daté autour des années 280-320, et celui des fibules de type Pröttel 3/4, daté des années 330/340 à 410. Si l'on ne retient que la datation typologique, on compte trois fibules de type 1 et cinq de type $3 / 4$. Or, la fibule $n^{\circ} 2$, de type 1, associée à des monnaies plus tardives, se rattache au groupe chronologique des fibules de type 3/4. Ainsi, la période 280-320 n'est plus représentée que par deux individus, alors que la période 330/340-410 en compte six. La datation de ces fibules indique donc une fréquentation militaire (ou officielle) importante à partir de l'époque constantinienne.

B. F. 


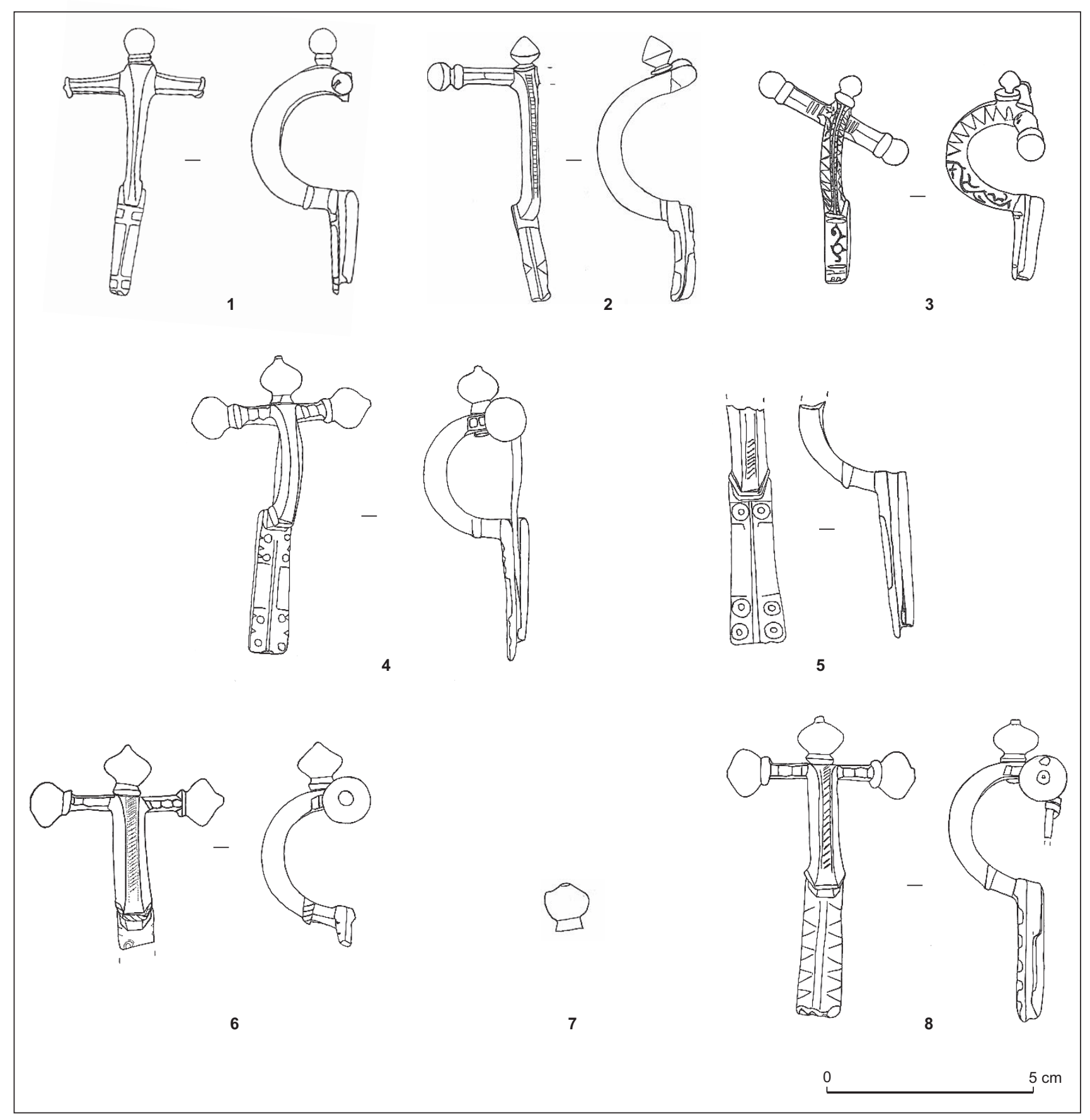

Fig. 36 - Fibules cruciformes en alliage cuivreux découvertes à Mandeure (dessins : I. Dard). 


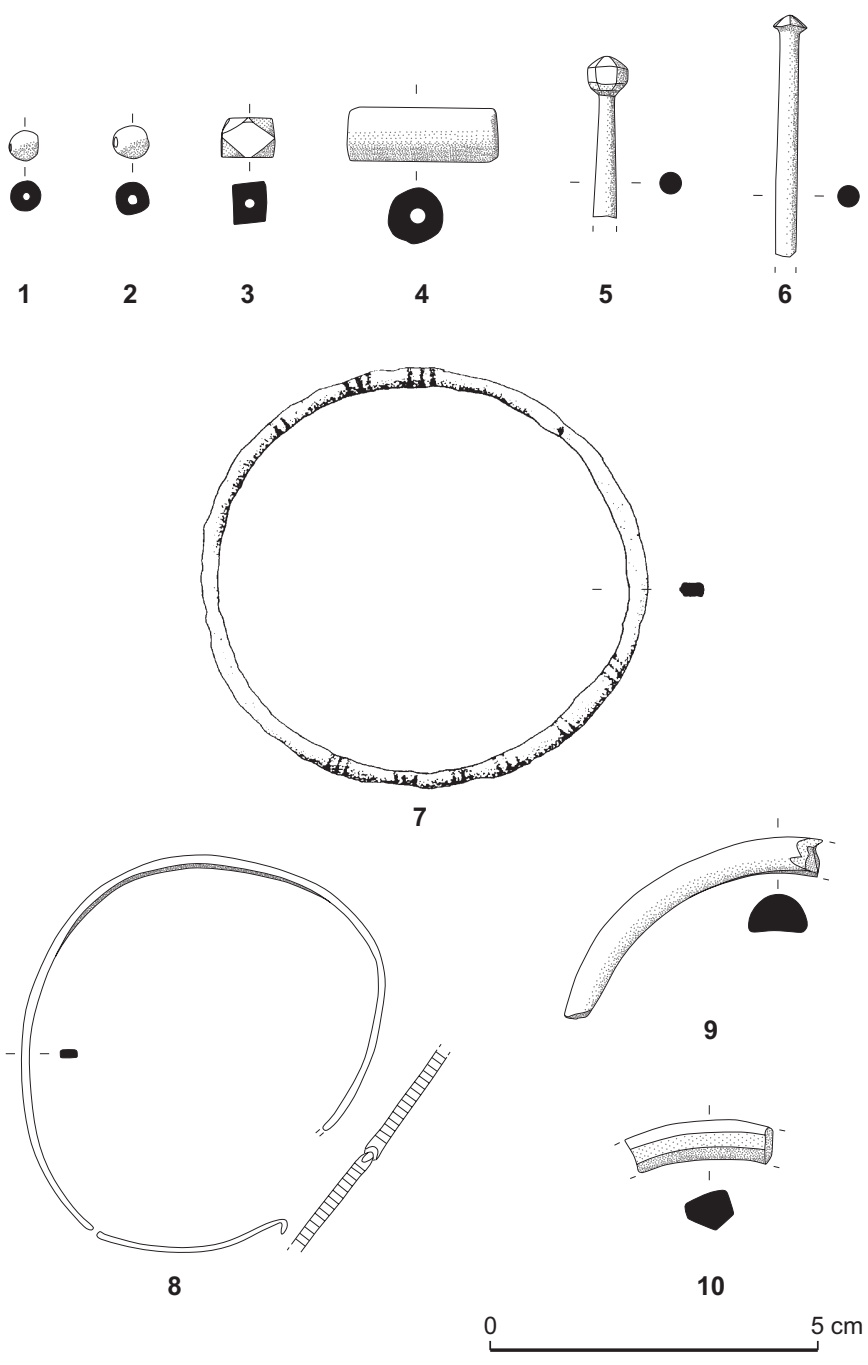

Fig. 37 - Objets de parure découverts à Mandeure : 1-4, perles en verre; 5, 6, épingles à cheveux en os; 7-8, bracelets en alliage cuivreux; 9, bracelet en verre; 10, bracelet en schiste (dessin : I. Dard).

\section{LA CÉRAMIQUE}

L'étude du mobilier céramique attribuable à l'Antiquité tardive ne permet d'approcher la chronologie d'occupation de la fortification que de façon sommaire (fig. 38). Corrélée à l'interprétation stratigraphique, l'étude céramologique permet d'inscrire les événements qui ponctuent la vie de la fortification dans une trame chronologique plus ou moins lâche. La construction de la fortification peut ainsi être située vers la fin du premier tiers $d u I^{\mathrm{e}} \mathrm{s}$. Les arguments concernant la durée de son occupation sont ténus et ceux concernant son abandon, plus encore.
S'il est certain qu'elle fut occupée pendant tout le $\mathrm{IV}^{\mathrm{e}} \mathrm{s}$., son existence au $\mathrm{V}^{\mathrm{e}} \mathrm{s}$. ne se lit plus qu'en filigrane. Ainsi, l'étude du mobilier céramique complète l'analyse des séries monétaires.

Dans le cadre du présent bilan, nous portons notre attention plus particulièrement sur l'identification des différentes importations céramiques. Cette approche met en lumière un faciès d'approvisionnement où la variété et l'exotisme des importations posent à la fois le problème de la situation géographique de Mandeure et celui de la nature de l'occupation de la fortification.

\section{LES PRODUCTIONS ARGONNAISES}

\section{Les sigillées tardives}

Leur présence est largement attestée. Le répertoire typologique est varié et bien illustré : il ne se restreint pas à la seule présence de Chenet 320, mais est aussi constitué des formes Chenet 301, 307, 313, 314 et $324{ }^{23}$.

La forme Chenet 320 est présente dans sa variante la plus précoce, la moins carénée, comme dans sa variante plus tardive, à profil plus massif et carène fortement marquée (Brulet, 1990, pl. 26). Les pâtes ne sont pas zonées.

Les molettes appartiennent principalement aux groupes 2 et 3 de W. Hübener ${ }^{24}$. Elles s'inscrivent dans un créneau chronologique axé sur le premier tiers du IV ${ }^{\mathrm{e}}$ s., défini par la phase 1 de Bayard et l'évolution typochronologique des groupes de W. Dijkman (Bayard, 1990 ; Dijkman, 1992).

L'ensemble de ces critères permet de définir une fourchette chronologique pour ces productions argonnaises, qui couvre la totalité du IV ${ }^{\mathrm{e}} \mathrm{s}$. Les éléments pour raisonner sur le $\mathrm{V}^{\mathrm{e}} \mathrm{s}$. sont plus ténus: les productions argonnaises se limitent principalement à la variante la plus tardive du Chenet 320 et à une coupelle Chenet 314. On note l'absence de molettes à motifs chrétiens. Elle peut être imputable à la nature de l'échantillon ou traduire des modifications dans les réseaux d'approvisionnement, car l'occupation du site $\mathrm{au} \mathrm{V}^{\mathrm{e}} \mathrm{s}$. est suggérée par la présence d'autres productions céramiques.

23. Pour la typologie de référence des productions d'Argonne, consulter les travaux de G. Chenet (1941, pl. 12-22). Cet ouvrage intègre et complète les premiers travaux de W. Unversagt publiés en 1916 (Unversagt, 1916). Pour un premier essai de regroupement typologique, se rapporter à l'ouvrage de W. Hübener (1968). La chronologie de ses groupes typologiques a été revue par W. Dijkman en 1992.

24. L'identification détaillée des molettes a été réalisée par P. Van Ossel. 

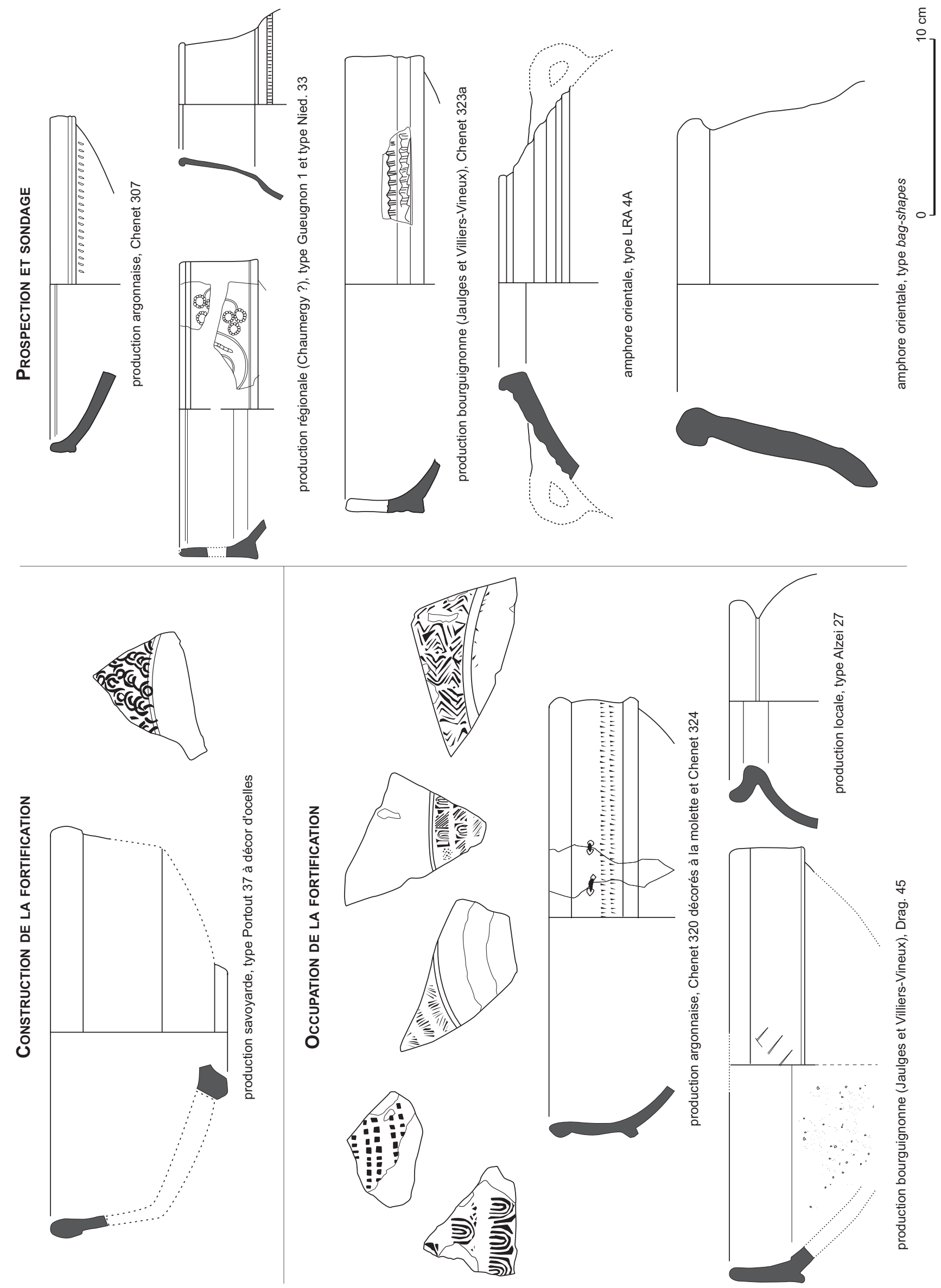


\section{LES PRODUCTIONS BOURGUIGNONNES}

\section{Les céramiques à revêtement argileux}

Deux centres de production ont été reconnus : Gueugnon (Saône-et-Loire), d'une part, et Jaulges et Villiers-Vineux (Yonne), d'autre part. Les productions de Gueugnon s'illus-trent à travers les jattes de type 1 (Creuzenet, 1996). Elles sont aussi produites dans l'atelier franc-comtois de Chaumergy (Charlier, 1996). Les exemplaires se caractérisent par une qualité d'engobe remarquable, présentant un grésage uniforme et des reflets métallescents. Si leur chronologie de production reste floue, on peut toutefois signaler qu'elles sont fréquentes dans des contextes de la première moitié du IV ${ }^{\mathrm{e}}$ s., comme à Escolives-Sainte-Camille (Yonne) et à Chevroches (Nièvre) (Mouton, 1996 ; Mouton-Venault, Devevey, 2005). Les productions de Jaulges et Villiers-Vineux sont bien représentées d'un point de vue quanti-tatif, mais le répertoire se restreint aux gobelets et à la jatte Chenet 323A.

Cette dernière connaît une évolution typochronologique depuis le début du IV ${ }^{\mathrm{e}} \mathrm{s}$. jusqu'au milieu du V $\mathrm{V}^{\mathrm{e}} \mathrm{s}$. (Séguier, Morize, 1996). Les exemplaires identifiés présentent un engobe de belle qualité, souvent métallescent, sans aucun zonage de la pâte. Ces différents critères caractérisent des productions qui circulent au cours et jusqu'à la fin du $\mathrm{IV}^{\mathrm{e}} \mathrm{s}$.

\section{LES PRODUCTIONS DES ATELIERS SAVOYARDS}

\section{Les céramiques à revêtement argileux}

Elles concurrencent directement les productions argonnaises. Seule une étude plus approfondie pourra permettre de mesurer la part réelle des différentes productions aux $\mathrm{IV}^{\mathrm{e}}$ et $\mathrm{V}^{\mathrm{e}} \mathrm{s}$. Elles s'illustrent à travers les formes Portout 1 , Portout 15, Portout 37 et Portout 75, et s'inscrivent dans un large créneau chronologique couvrant le $\mathrm{IV}^{\mathrm{e}} \mathrm{s}$. et la première moitié du $\mathrm{V}^{\mathrm{e}} \mathrm{s}$.

\section{LES PRODUCTIONS DE PROVENANCES VARIÉES ET « RÉGIONALES »}

\section{Les céramiques rugueuses}

Cette appellation regroupe des critères techniques et typologiques: elle englobe des productions de provenance variée (Eiffel, Argonne, régionale, etc.). Cette diversité de provenance s'illustre sur le site par une importante diversité de pâtes. Une étude plus approfondie permettra de distinguer les différentes productions (régionales ou importées). La présence de la forme Alzei 27, datable de la seconde moitié $d u \mathrm{IV}^{\mathrm{e}} \mathrm{s}$. et du premier tiers du V $\mathrm{e}$. (Brulet, 1990, p. 431, pl. 27), est récurrente.

\section{Les céramiques à revêtement argileux}

On constate la présence récurrente de mortiers dits «rhétiques»; cette appellation regroupe des productions à revêtement argileux provenant de Suisse (Rhétie I). Elles présentent un engobe interne et externe rouge vif, de belle qualité, et sont datables du $\mathrm{III}^{\mathrm{e}} \mathrm{s}$. (Schucany, 1999, fig. 3, $\mathrm{n}^{\mathrm{o}} 8$, p. 338). La majorité des productions regroupées sous cette appellation et reconnues sur le site est apparentée à cette production d'un point de vue typologique, mais présente un aspect différent : l'engobe est brun orangé et la nature de leur pâte ne les distingue en rien des productions à revêtement argileux régionales. Ces productions ont été identifiées dans des contextes du deuxième tiers du IV ${ }^{\mathrm{e}} \mathrm{s}$. à Langres en Haute-Marne (Joly et al., 2001).

\section{LES IMPORTATIONS AFRICAINES ET ORIENTALES}

\section{Les sigillées Claires D, africaines}

Quelques fragments de sigillée Claire D attestent la présence d'importations africaines. Ce type d'importation reste rare au-delà de la vallée du Rhône où il n'est attesté que dans des contextes du V ${ }^{\text {e }}$ s. ${ }^{25}$.

Un fragment de lampe a également été identifié, sans qu'il soit possible de déterminer s'il s'agit d'une production africaine ou orientale.

\section{Les amphores orientales}

Trois individus ont été identifiés : une amphore LRA 4, dans sa variante la plus précoce (A1) (Pieri, 1998, p. 101), un petit fragment de LRA 1 et une amphore de type back-shaped.

La présence d'amphores orientales constitue un fait à souligner. Sans être exceptionnelle, la LRA 4 reste un mobilier peu courant dans les contextes du nord de la Gaule. Elle est toutefois, avec la LRA 1, parmi celles qui pénètrent le plus fréquemment au-delà de la vallée du

25. Ces importations sont encore présentes à Mâcon en Saône-et-Loire (Saulnier, 1988). 
Rhône. L'importation de la LRA 4 débute dès le milieu du $I^{\mathrm{e}} \mathrm{s}$. dans des contextes méditerranéens, mais son importation massive se fait à partir du début du $\mathrm{V}^{\mathrm{e}} \mathrm{s}$. jusqu'au $\mathrm{VI}^{\mathrm{e}} \mathrm{s}$. (Pieri, 1998, p. 105-106).

La présence d'une amphore de type back-shaped est plus surprenante. À notre connaissance, elle reste exceptionnelle, mais il est vrai qu'il s'agit d'un mobilier difficile à identifier.

\section{SYNTHÈSE}

La variété des approvisionnements céramiques témoigne d'un certain dynamisme des échanges commerciaux à la fois tournés vers le nord de la Gaule (présence des productions dites « rugueuses », argonnaises ou bourguignonnes) et le sud (ateliers savoyards), voire le monde méditerranéen (importations africaines et orientales). Ce phénomène s'explique en partie par la situation géographique privilégiée de Mandeure-Mathay, sur un axe de circulation Rhône-SaôneRhin. Il semble toutefois important de souligner la présence des amphores orientales, lampes et sigillées Claires D qui ne sont pas monnaie courante sur les habitats de Gaule septentrionale, qu'il s'agisse des sites suisses (comme celui de Sion, par exemple : Schucany et al., 1999) ou bourguignons. Reste à savoir si cet approvisionnement singulier relève de la présence des militaires ou d'une position stratégique de Mandeure, au sein d'un réseau commercial.

La variété des productions est par ailleurs un atout pour raisonner sur la chronologie du site: elle permettra de croiser différents fossiles directeurs de l'Antiquité tardive, utilisés soit pour le nord de la Gaule (Argonne décorée à la molette, jatte Chenet 323A), soit pour le sud (productions des ateliers savoyards, productions africaines et orientales), et ainsi, d'affiner le créneau chronologique.

S. M.

\section{CONGLUSION}

Même s'il reste de nombreuses inconnues concernant la structuration interne de la fortification du Bas-Empire de Mandeure, les recherches récentes, couplées aux données plus anciennes, permettent de dresser un premier bilan qui place Mandeure parmi les sites majeurs du dispositif militaire de la Maxima Sequanorum (fig. 39). Rappelons que l'édification de la fortification modifie considérablement la trame urbaine de l'agglomération antique. Sa construction implique notamment la fin de la circulation sur l'importante voie nord-est/sud-ouest qui reliait la zone urbanisée située dans la boucle du Doubs au gué près du sanctuaire. L'emploi de spolia provenant des édifices publics témoigne également des changements intervenus dans la topographie de la ville.

En l'état actuel de la recherche, la topographie et le plan de la fortification semblent se dessiner de manière plus précise (fig. 40). L'enceinte a la forme d'un demi-cercle dont le segment rectiligne s'appuie au Doubs. La partie incurvée est munie de tours semi-circulaires saillantes. À partir des deux tours attestées, on peut supposer l'existence d'au moins quatre tours intermédiaires. La partie rectiligne, située côté Doubs, semble dépourvue de tours intermédiaires. Aux jonctions entre la partie courbe et la partie rectiligne, on peut s'attendre à trouver des tours d'angle. Par ailleurs, la fortification devait avoir deux portes, situées sur son axe perpendiculaire au Doubs, l'une au milieu du tronçon rectiligne et l'autre au milieu du tronçon courbe. Quant aux dimensions, l'axe perpendiculaire (entre les deux portes supposées) mesure $115 \mathrm{~m}$ et l'extension maximale de la fortification - parallèlement au Doubs - approximativement $165 \mathrm{~m}$.

La fortification de Mandeure présente sur le plan typologique de fortes similitudes avec celle de Soleure en Suisse (Spycher, Schucany, 1997, fig. 90). Elles ont toutes deux la forme d'une cloche qui s'appuie sur un cours d'eau navigable (fig. 40). De plus, elles possèdent des dimensions comparables, Soleure étant légèrement plus petite avec ses $150 \mathrm{~m}$ le long de l'Aare et ses $120 \mathrm{~m}$ pour l'axe perpendiculaire.

Quelques distinctions peuvent toutefois être remarquées entre ces deux ouvrages. La profondeur des fondations de la fortification de Soleure n'est que de $1 \mathrm{~m}$, alors qu'elle atteint près de $2 \mathrm{~m}$ à Mandeure. D'autre part, l'épaisseur du mur d'enceinte de Soleure est comprise entre $2 \mathrm{~m}$ et 2,50 m, en ce qui concerne son élévation (Spycher, Schucany, 1997, p. 107 et p. 160). Pour le cas de Mandeure, on peut estimer, en se basant sur la largeur des fondations, que le mur d'enceinte avait au minimum entre 2,5 m et $3 \mathrm{~m}$ d'épaisseur.

À Mandeure, on ne peut que regretter l'absence d'élévation, mais on imagine facilement une enceinte massive et relativement haute, grâce aux dimensions importantes des fondations. Pour comparaison, le mur d'enceinte de Soleure est encore conservé sur $9 \mathrm{~m}$ de haut (Spycher, Schucany, 1997, p. 160). Les tours de la fortification de Mandeure devaient également être imposantes puisqu'elles s'élevaient sur des fondations saillantes de $4 \mathrm{~m}$ et larges de $8 \mathrm{~m}$. Par ailleurs, il est nécessaire de souligner le soin apporté à la mise en place de la semelle de fondation. Les nombreux piquets sont disposés de façon régulière. Les strates du hérisson en pierres sèches ont été soigneusement aménagées. L'emploi de spolia témoigne, en outre, d'une bonne gestion des matériaux de démolition. 

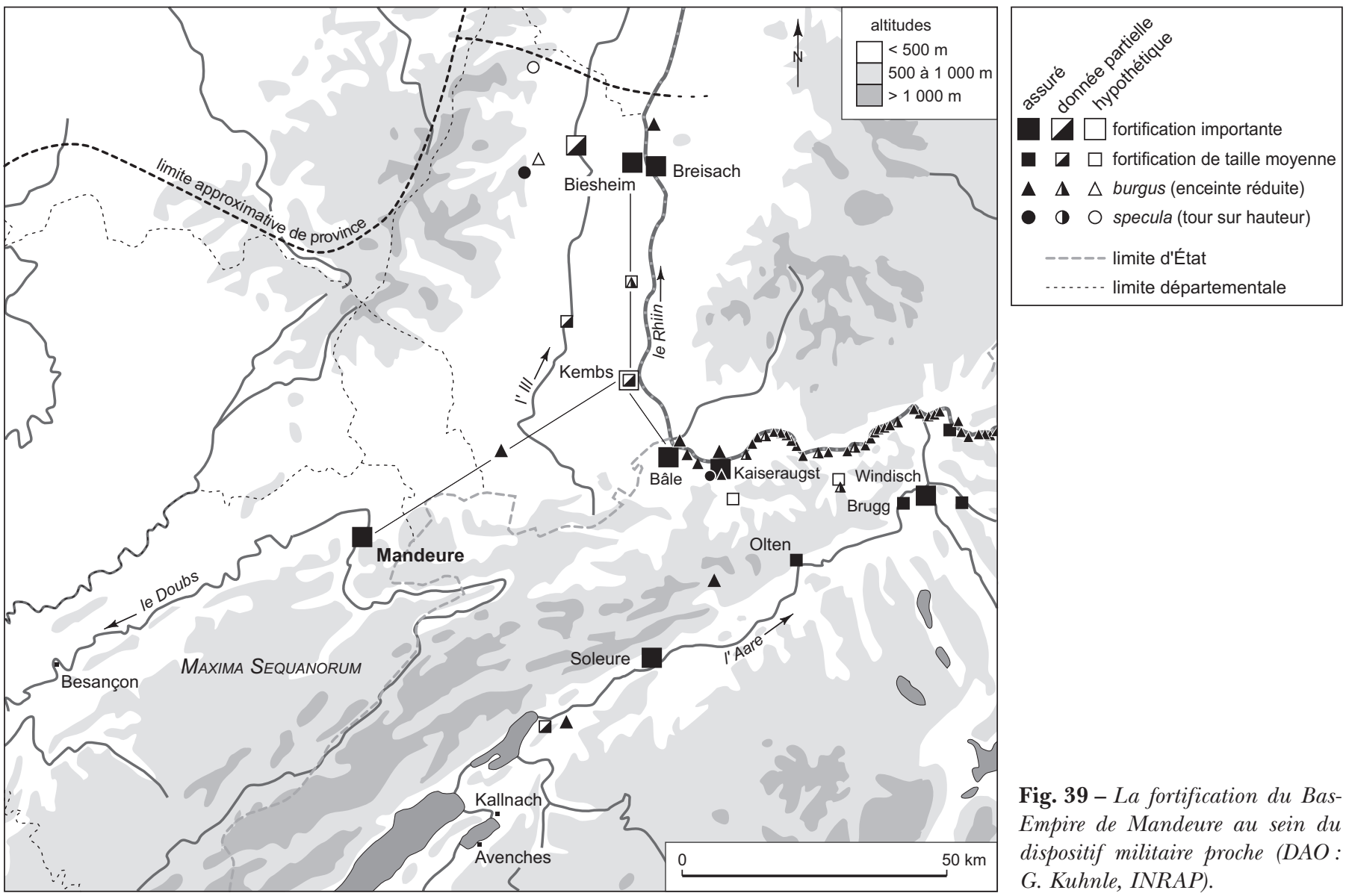

Fig. 39 - La fortification du BasEmpire de Mandeure au sein du dispositif militaire proche (DAO: G. Kuhnle, INRAP).
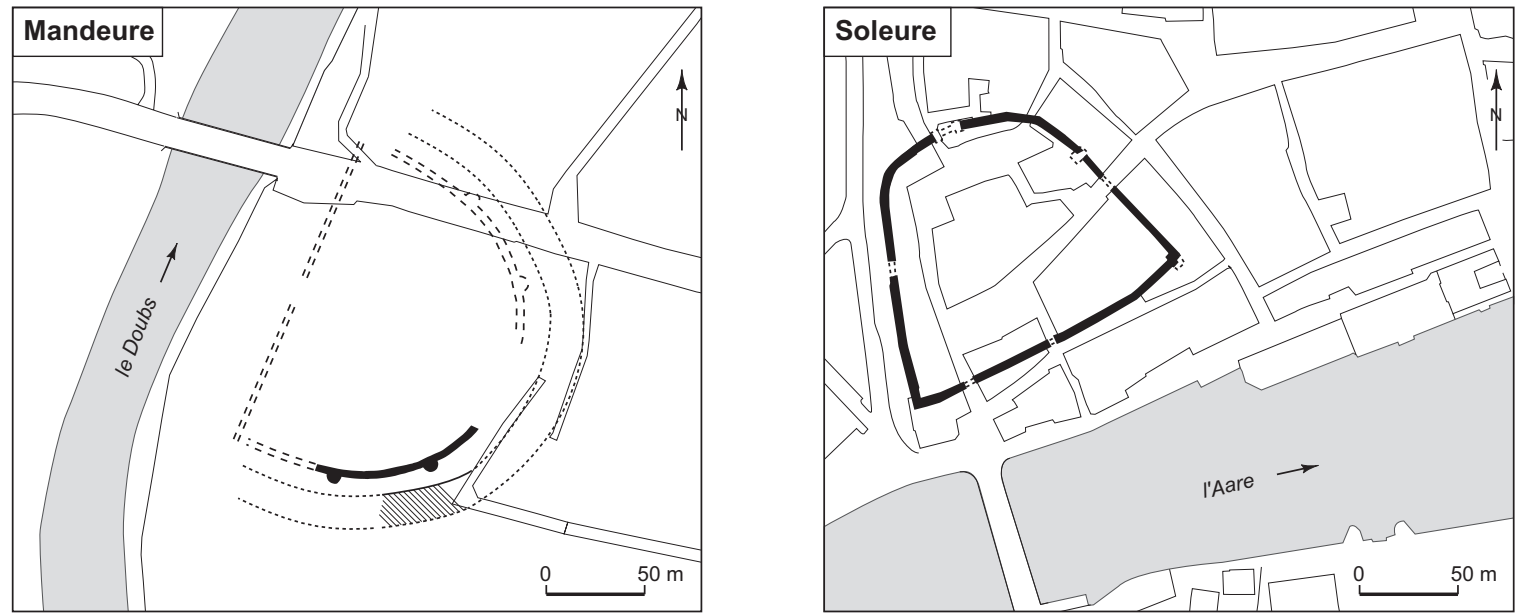

Fig. 40 - Fortifications du Bas-Empire (DAO Mandeure : G. Kuhnle et B. Fort, INRAP ; Soleure, d'après Spycher, Schucany, 1997). 
Grâce aux fouilles récentes et aux recherches associées, notamment l'analyse monétaire, la chronologie du site a également pu être précisée. En effet, on constate un pic monétaire sur le site entre 330 et 348 (fin du règne de Constantin et règne de Constant en Occident). L'étude de la céramique, voire celle des petits objets, montre un phénomène similaire: la période constantinienne est la mieux représentée sur la fortification de Mandeure.

On peut alors penser que c'est à cette période que la fortification est fondée. Au vu de l'ampleur et de la qualité de sa construction, il faut supposer qu'elle a été érigée à l'initiative d'une autorité forte, vraisemblablement impériale, à l'instar de ce que propose C. Schucany pour la fortification de Soleure, construite à la même époque (Spycher, Schucany, 1997, p. 157 et p. 165). L'hypothèse que la fortification de Mandeure soit l'œuvre de militaires est par ailleurs étayée par la présence de militaria et de quatre briques estampillées de la légion IMartia (provenance indéterminée sur les communes de Mandeure et Mathay) (fig. 41). La grande variété des importations céramiques suggère également la présence d'un détachement de militaires sur la fortification.

Avec la période valentinienne, l'occupation de la fortification connaît une nette reprise. Elle est reflétée par le mobilier qui est moins abondant que celui de l'époque constantinienne mais tout aussi varié. Ce matériel ne constitue pas de facteur discriminant pour identifier la population, qui peut être civile, militaire ou mixte. Il nous paraît fort probable que les militaires aient régulièrement fréquenté le site durant la période valentinienne, comme ils l'ont certainement fait dans la première moitié $d u \mathrm{IV}^{\mathrm{e}} \mathrm{s}$.

Le sort de la fortification $\mathrm{au} \mathrm{V}^{\mathrm{e}} \mathrm{s}$. et au-delà reste encore très flou. Le démantèlement systématique de son enceinte, tombée en ruine, ne semble être intervenu, d'après les éléments céramiques recueillis, qu'aux $\mathrm{XIV}^{\mathrm{e}}-\mathrm{XV}^{\mathrm{e}} \mathrm{s}$.

G. K., B. F., A. B.

\section{CONCLUSION}

Les recherches menées entre 2001 et 2004 confirment le rôle éminent de l'agglomération d'Epomanduodurum au sein de la Séquanie, et plus largement du nord-est des Gaules, depuis l'époque gauloise jusqu'au Bas-Empire. L'organisation du quartier cultuel, sa place au sein de la trame urbaine, son rôle dans la genèse de l'agglomération, se précisent peu à peu.

Le développement au Haut-Empire d'un complexe monumental s'inscrit en effet dans un processus initié à la fin de l'âge du Fer, sans doute dès le III $^{\mathrm{e}} \mathrm{s}$. av. J.-C., avec la création
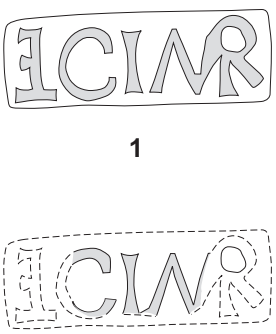

3
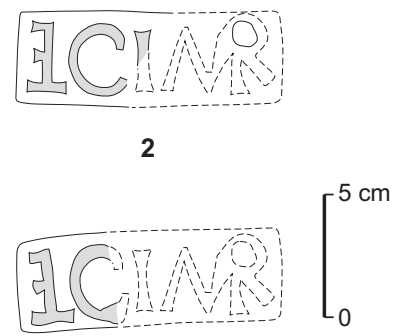

Fig. 41 - Estampilles relevées sur les briques provenant de Mandeure (dessin : I. Dard).

d'un grand sanctuaire laténien. Si les vestiges d'habitat de la même période restent encore largement dans l'ombre, on dispose à présent d'indices suffisamment nombreux et cohérents pour que l'existence d'une agglomération gauloise associant zone d'habitat, sanctuaire et nécropole, remarquablement située au contact de la vallée du Doubs et du fossé rhénan, dépasse le stade de la simple hypothèse.

Jusqu'à ces dernières années, le sanctuaire et le théâtre antiques pouvaient sembler isolés, en limite sud de la boucle du Doubs. Cette idée est remise en cause par les avancées concernant la topographie de la ville antique. En réalité, le complexe monumental dont l'organisation se dessine peu à peu occupe une position clé, à la conjonction de deux axes de circulation majeurs, et s'insère remarquablement dans la trame urbaine.

Le grand sanctuaire gallo-romain exhumé au $\mathrm{XIX}^{\mathrm{e}} \mathrm{s}$. reste en grande partie énigmatique, faute de données nouvelles. En revanche, la connaissance du théâtre bénéficie d'avancées significatives. Les dimensions exceptionnelles de l'édifice vont de pair avec un traitement très classique des façades et de l'ornementation, et un apparat décoratif qui n'a rien à envier à celui des édifices comparables d'Avenches ou Augst. Ainsi se dessinent un schéma urbanistique et un programme architectural cohérents, qui supposent des projets édilitaires de grande ampleur. Faute de mentions épigraphiques, nous ignorons pour l'instant les noms des riches familles séquanes à l'origine de cette réussite et l'arrière-plan historique dans lequel elle s'inscrit. Les recherches en cours et celles à venir devraient en revanche permettre de préciser les rythmes et modalités du développement de l'agglomération au Haut-Empire, ainsi que les mutations qu'elle connaît au Bas-Empire, grâce à l'accentuation des études portant sur les édifices publics d'une part, à l'intensification des investigations pluridisciplinaires à l'échelle de la ville et de son territoire, d'autre part.

P. B. 